\title{
Exosomes Exploit the Virus Entry Machinery and Pathway To Transmit Alpha Interferon-Induced Antiviral Activity
} \author{
Lu Peng, Jianhua Li, ${ }^{a}$ Zhenghong Yuan ${ }^{a}$ \\ aKey Laboratory of Medical Molecular Virology, School of Basic Medical Sciences, Shanghai Medical College of \\ Fudan University, Shanghai, China \\ bShanghai Public Health Clinical Center, Shanghai Medical College of Fudan University, Shanghai, China
}

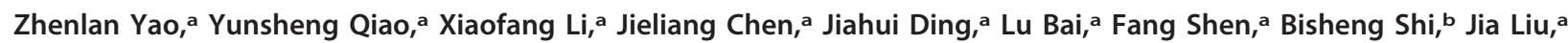

ABSTRACT Alpha interferon (IFN- $\alpha$ ) induces the transfer of resistance to hepatitis B virus (HBV) from liver nonparenchymal cells (LNPCs) to hepatocytes via exosomes. However, little is known about the entry machinery and pathway involved in the transmission of IFN- $\alpha$-induced antiviral activity. In this study, we found that macrophage exosomes uniquely depend on $\mathrm{T}$ cell immunoglobulin and mucin receptor 1 (TIM-1), a hepatitis A virus (HAV) receptor, to enter hepatocytes for delivering IFN- $\alpha$ induced anti-HBV activity. Moreover, two primary endocytic routes for virus infection, clathrin-mediated endocytosis (CME) and macropinocytosis, collaborate to permit exosome entry and anti-HBV activity transfer. Subsequently, lysobisphosphatidic acid (LBPA), an anionic lipid closely related to endosome penetration of virus, facilitates membrane fusion of exosomes in late endosomes/multivesicular bodies (LEs/MVBs) and the accompanying exosomal cargo uncoating. Together, our findings provide comprehensive insights into the transmission route of macrophage exosomes to efficiently deliver IFN- $\alpha$-induced antiviral substances and highlight the similarities between the entry mechanisms of exosomes and virus.

IMPORTANCE Our previous study showed that LNPC-derived exosomes could transmit IFN- $\alpha$-induced antiviral activity to HBV replicating hepatocytes, but the concrete transmission mechanisms, which include exosome entry and exosomal cargo release, remain unclear. In this study, we found that virus entry machinery and pathway were also applied to exosome-mediated cell-to-cell antiviral activity transfer. Macrophage-derived exosomes distinctively exploit hepatitis A virus receptor for access to hepatocytes. Later, CME and macropinocytosis are utilized by exosomes, followed by exosome-endosome fusion for efficient transfer of IFN$\alpha$-induced anti-HBV activity. We believe that understanding the cellular entry pathway of exosomes will be beneficial to designing exosomes as efficient vehicles for antiviral therapy.

KEYWORDS IFN- $\alpha$, TIM-1, endocytosis, exosome, hepatitis B virus

$\mathrm{H}$ epatitis B virus (HBV) is a small, enveloped DNA virus that replicates via an RNA intermediate and belongs to the Hepadnaviridae family (1). Approximately 400 million people are chronically infected with HBV worldwide (2). Chronic HBV infection is a major risk factor for the development of liver cirrhosis and hepatocellular carcinoma (3). Alpha interferon (IFN- $\alpha$ ) is licensed for the treatment of HBV chronic infection, with a response rate of $30 \%$ to $40 \%$ and a clinical cure rate of approximately $10 \%$ (4); however, the efficacy of IFN- $\alpha$ is limited due to inhibition by viral proteins $(5,6)$. It remains to be further elucidated how IFN- $\alpha$ achieves therapeutic effects in chronic HBV patients through direct antiviral effects or indirect modulation of antiviral response (7). We and others previously reported that IFN- $\alpha$ induced the transfer of resistance to

Received 6 September 2018 Accepted 10 September 2018

Accepted manuscript posted online 3 October 2018

Citation Yao Z, Qiao Y, Li X, Chen J, Ding J, Bai L, Shen F, Shi B, Liu J, Peng L, Li J, Yuan Z. 2018 Exosomes exploit the virus entry machinery and pathway to transmit alpha interferoninduced antiviral activity. J Virol 92:e01578-18. https://doi.org/10.1128/JVI.01578-18.

Editor Rozanne M. Sandri-Goldin, University of California, Irvine

Copyright $\odot 2018$ American Society for Microbiology. All Rights Reserved.

Address correspondence to Jianhua Li, jianhuali@fudan.edu.cn, or Zhenghong Yuan, zhyuan@shmu.edu.cn.

Z.Y. and Y.Q. contributed equally to this article. 
hepatitis viruses from nonpermissive liver nonparenchymal cells (LNPCs), including liver resident macrophages, to permissive hepatocytes via exosomes, but the underlying mechanism remains largely unclear (8-12).

Exosomes are 40 - to $100-\mathrm{nm}$ membrane vesicles derived from the intraluminal vesicles (ILVs) of multivesicular bodies (MVBs) that are secreted into the extracellular milieu through the fusion of MVBs with the plasma membrane $(13,14)$. These vesicles can serve as mediators of intercellular communication to exchange functional proteins, lipids, mRNAs, and microRNAs (miRNAs) among cells (15-17). Given the emerging roles of exosomes from IFN- $\alpha$-induced LNPCs in the antiviral innate response and their therapeutic potential $(8,9,18,19)$, it is important to understand the molecular mechanisms by which nonparenchymal cell-derived exosomes are taken up into hepatocytes and release their cargo to inhibit HBV replication.

The entry strategy used by a given exosome may depend on the proteins and lipids on the surfaces of both exosomes and recipient cells (20-22). The routes and fates of exosome internalization may partially overlap those of the virus $(10,23,24)$. In this study, we found that the hepatitis A virus (HAV) receptor, T cell immunoglobulin and mucin receptor 1 (TIM-1), mediated the internalization of macrophage-derived exosomes into hepatocytes; we showed that the rapid clathrin-dependent pathway and sustained macropinocytosis, two primary pathways for virus invasion, were also used as the major endocytic routes for exosome entry and the transmission of IFN- $\alpha$-induced $\mathrm{HBV}$ resistance. After internalization, membrane fusion of exosomes and accompanying exosomal cargo uncoating took place in late endosomes (LEs)/MVBs, relying on the LE-specific anionic lipid lysobisphosphatidic acid (LBPA). Collectively, our findings demonstrate that macrophage exosomes require virus entry machinery and pathway for transmission of IFN- $\alpha$-induced antiviral activity to combat HBV in hepatocytes.

(This article was submitted to an online preprint archive [25].)

\section{RESULTS}

PtdSer receptor TIM-1 uniquely mediates exosome entry and transfer of IFN$\boldsymbol{\alpha}$-induced anti-HBV activity. Exosomes were isolated from the culture of THP-1derived macrophages by differential centrifugation, as described previously (8). Membrane vesicles approximately $100 \mathrm{~nm}$ in diameter with a cup-shaped structure typical of exosomes were identified by electron microscopy (Fig. 1A). Further characterization by immunoblotting indicated the presence of exosomal markers (CD63, TSG101, and Alix) and conserved exosomal proteins (LAMP-2 and $\beta$-actin) and the absence of the endosomal marker EEA1 (Fig. 1B). Isolated exosomes were labeled with the fluorescent lipid dye PKH26. We observed the internalization of PKH26-labeled exosomes by hepatocyte-derived HepG2 cells, which were stained for cytoskeletal F-actin with phalloidin-iFluor 488 (Fig. 1C) at $37^{\circ} \mathrm{C}$, and found that the uptake kinetics were time and concentration dependent (data not shown).

PtdSer-an apoptosis marker typically located on the inner leaflet of the plasma membrane-is found on the outer membrane of exosomes from bone marrow-derived dendritic cells (BMDCs) and oligodendrocytes $(21,26)$. Previous experimental evidence indicates that some viruses may exploit PtdSer as an apoptotic disguise and enter target cells through PtdSer receptor-mediated internalization (27). We confirmed PtdSer expression on the outer membrane of macrophage-derived exosomes through annexin $\mathrm{V}$ labeling of exosomes isolated from macrophages (Fig. 1D).

To determine whether PtdSer receptors play roles in the entry of macrophagederived exosomes into hepatocytes, we then inhibited the expression of two hepatic PtdSer receptors involved in virus entry (27), T cell immunoglobulin and mucin receptor 1 (TIM-1) (Fig. 1E) and growth arrest-specific 6 (GAS6) (data not shown), in HepG2 cells with specific small interfering RNAs (siRNAs). The uptake of PKH26-labeled exosomes was significantly reduced in HepG2 cells after TIM-1 knockdown (Fig. 1F and G), but interference with GAS6 expression had no effect on exosome uptake (data not shown). It is notable that the IgV in ectodomains of TIM proteins binds PtdSer on viral envelope and enhances hepatitis A virus (HAV) entry 
A

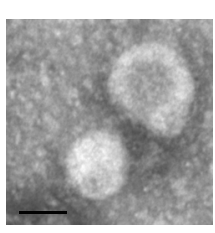

E

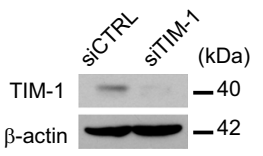

G

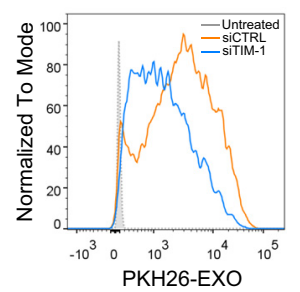

J

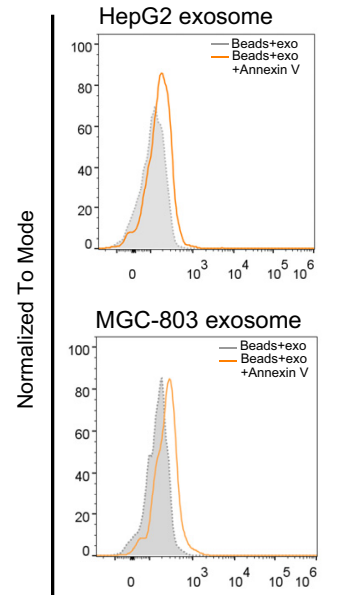

B

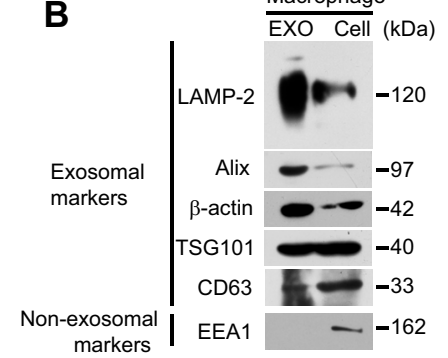

F

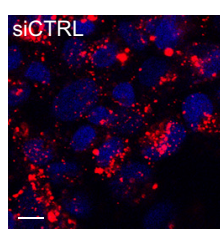

C

PKH26-EXO Actin filaments

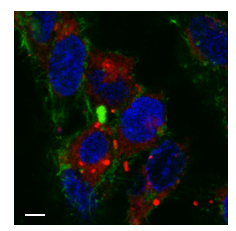

H
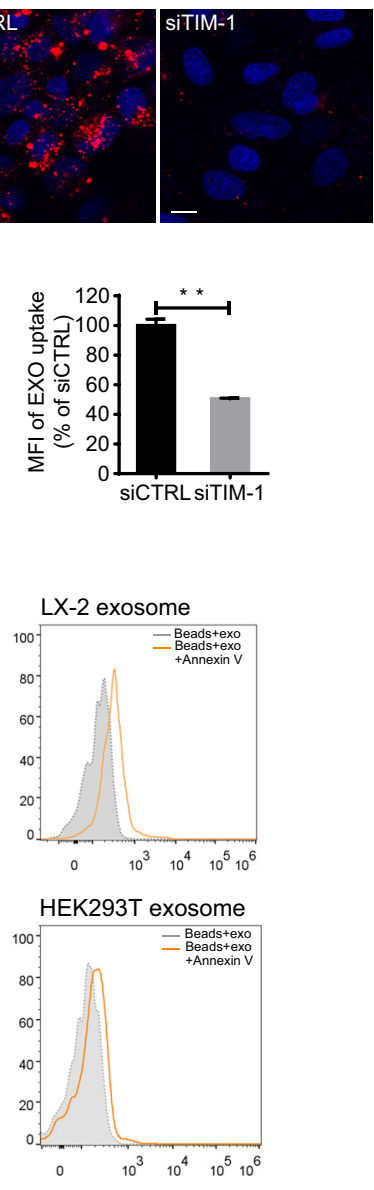

Annexin V-FITC

L

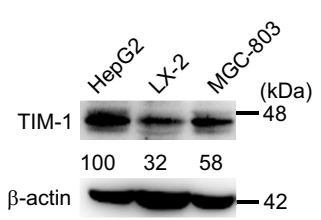

M

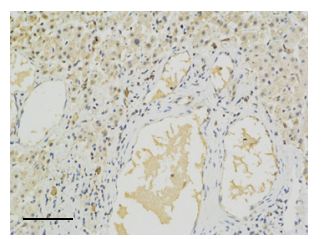

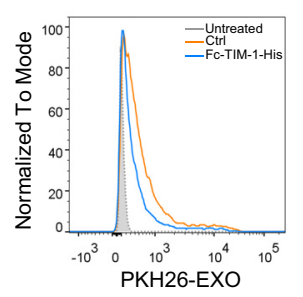

I
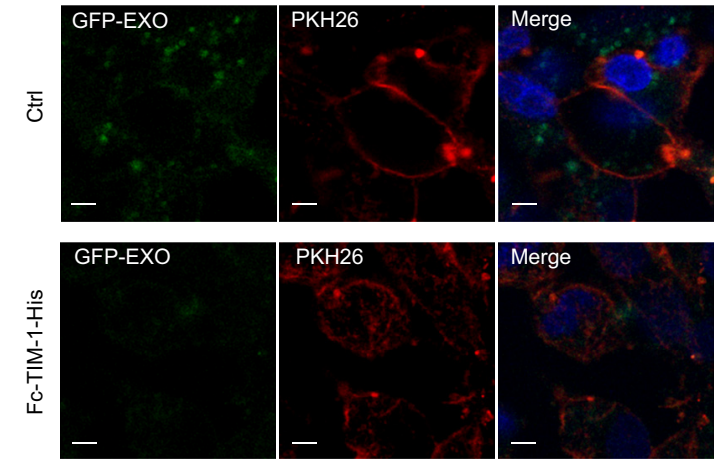

Merge

K
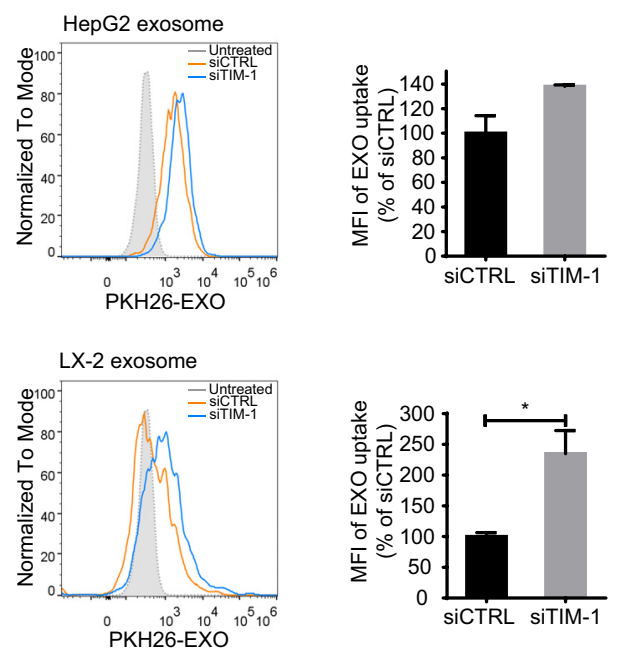

FIG 1 (Continued)

(28). We found that exogenous TIM-1 ectodomains competitively inhibited exosome internalization by HepG2 cells (Fig. 1H). Furthermore, preincubating green fluorescent protein (GFP)-carrying exosomes which were isolated from GFP-expressing macrophages with exogenous TIM- 1 ectodomains could hinder exosome binding to hepatocytes (Fig. 1I). These results suggested that TIM-1 is a functional receptor for the entry of macrophage exosomes into hepatocytes. 
$\mathbf{N}$
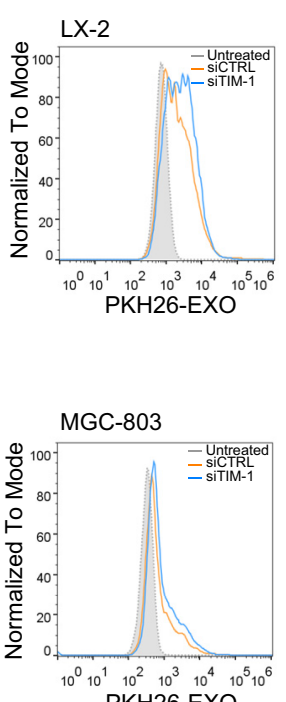

PKH26-EXO
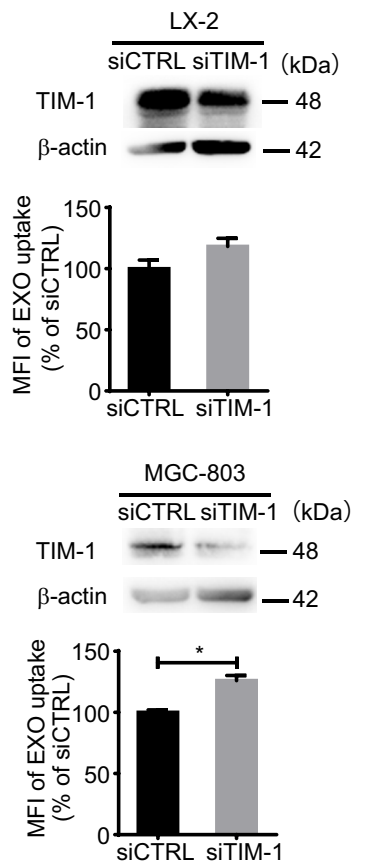

0
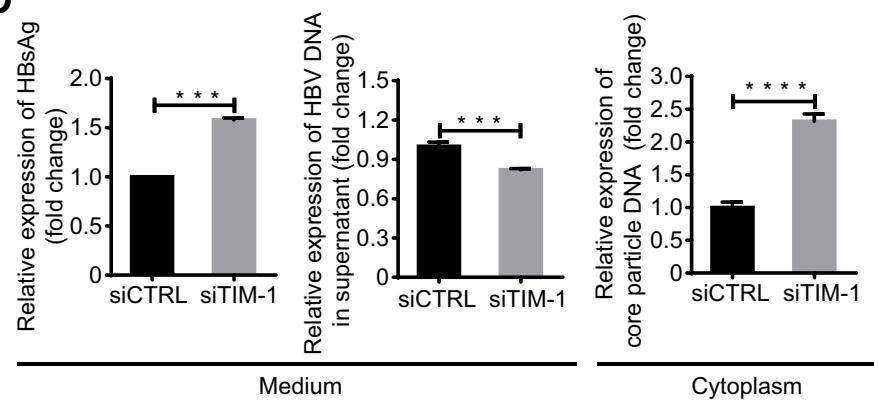

$\mathbf{P}$

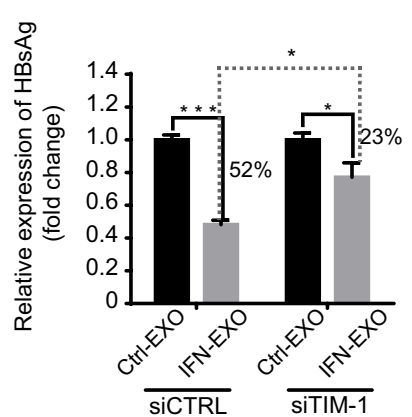

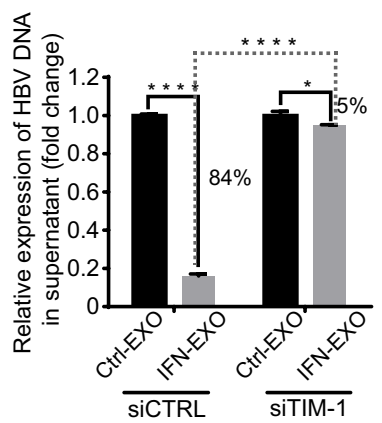

FIG 1 TIM-1 mediates exosome internalization and IFN- $\alpha$-induced anti-HBV activity transmission. (A) Electron microscopy of purified exosomes from macrophages. Scale bar: $100 \mathrm{~nm}$. (B) Immunoblot analysis of macrophage-derived exosomes (left) and corresponding cells (right) for exosomal and nonexosomal markers. (C) PKH26-labeled exosome internalization by HepG2 cells. Scale bar: $5 \mu \mathrm{m}$. (D) PtdSer detection on the macrophage exosome surface. Exosomes coating 4- $\mu \mathrm{m}$ latex beads were either stained or not with annexin V-FITC and analyzed by flow cytometry. (E) Knockdown validation of TIM-1 by immunoblotting. ( $F$ and G) Confocal images (F) or flow cytometry analysis (G) of PKH26-labeled exosome internalization by HepG2 cells after TIM-1 knockdown. Scale bars: $10 \mu \mathrm{m}$. For flow cytometry analysis, both histogram graph (left) and mean fluorescence intensities (MFI) (right) normalized to control siRNA (siCTRL)-transfected cells are presented. (H) Flow cytometry analysis of PKH26-labeled exosome internalization by HepG2 cells in the presence or absence (Ctrl) of Fc-TIM-1-His. MFI (right) is normalized to that of Ctrl cells. (I) Binding of GFP-exosomes preincubated with or without Fc-TIM-1-His to HepG2 cells with plasma membrane stained by PKH26. Scale bars: $5 \mu$ m. (J) PtdSer detection on the surfaces of exosomes derived from HepG2, LX-2, MGC-803, and HEK293T cells. (K) Flow cytometry analysis of PKH26-labeled HepG2 or LX-2 exosome internalization by HepG2 cells after TIM-1 knockdown. (L) Comparison of TIM-1 expression among HepG2, LX-2, and MGC-803 cells by immunoblotting. The expression of TIM-1 was normalized against $\beta$-actin and is presented (as percent) relative to its expression in HepG 2 cells. (M) Detection of TIM-1 (brown staining) in normal human liver section by IHC. (N) Flow cytometry analysis of PKH26-labeled macrophage exosome internalization by LX-2 or MGC-803 cells after TIM-1 knockdown. The TIM-1 knockdowns were validated by immunoblotting. (O) HepG2.2.15 cells were transfected with either siTIM-1 or siCTRL. The level of HBsAg in the culture medium (supernatant) was detected by ELISA. HBV DNA in the culture medium and intracellular core particle DNA were quantified by qPCR. (P) Blockade of IFN- $\alpha$-induced anti-HBV activity transmission by TIM-1 knockdown. HepG2.2.15 cells transfected with either siTIM-1 or siCTRL were treated with exosomes from IFN- $\alpha$-stimulated macrophages (IFN-EXO) or unstimulated cells (Ctrl-EXO). HBsAg and HBV DNA levels in the medium were measured by ELISA or quantified by qPCR. The error bars indicate the SD. ${ }^{*}, P<0.05 ;{ }^{* *}, P<0.01 ;{ }^{* *}, P<0.001 ;{ }^{* * * *}, P<0.0001$ (Student's $t$ test). The data are representative of those from three independent experiments.

We then asked whether TIM-1-mediated entry in hepatocytes was specific to macrophage exosomes. PtdSer was widely detected on the surface of exosomes from other cell types, including hepatic stellate cells (LX-2), hepatocytes (HepG2), embryonic kidney cells (HEK293T), and gastric adenocarcinoma cells (MGC-803) (Fig. 1J). Uptakes of these exosomes by HepG2 cells with TIM-1 knocked down were analyzed to determine whether TIM-1 also mediates the entry of exosomes from other cells. However, knocking down TIM-1 did not reduce the uptake of HepG2 exosomes and conversely enhanced the internalization of LX-2 exosomes (Fig. 1K). In addition, exosomes from nonhepatic cells, including HEK293T and MGC-803 cells, hardly enter hepatocytes (data not shown). Next, we investigated if TIM-1 was required for macrophage exosome entry in other cell types. TIM-1 was relatively highly expressed in HepG2 cells compared to LX-2 and MGC-803 cells (Fig. 1L). A human liver section also showed that hepatocytes were specifically abundant in TIM-1 (Fig. 1M). Interfering with TIM-1 expression in LX-2 and MGC-803 cells did not inhibit macrophage exosome uptake (Fig. 1N). These results ruled out TIM-1 as a universal factor for macrophage exosome entry and suggested that TIM-1-mediated exosome entry might be unique to macrophage exosomes and hepatocytes. 
The above-described results bring up the question whether highly expressed TIM-1 was necessary for IFN- $\alpha$-induced anti-HBV activity transfer to hepatocytes by macrophage exosome. To answer the question, we studied the exosome-mediated anti-HBV effect in HepG2.2.15 cells with TIM-1 interference. However, it was surprising that downregulating TIM-1 expression moderately increased HBsAg in the culture medium and slightly decreased HBV DNA in the culture medium, while the intracellular core particle-associated DNA was greatly upregulated (Fig. 10), suggesting that TIM-1 might play multiple roles in the HBV life cycle. Despite these unexpected effects, we still found that IFN- $\alpha$-induced anti-HBV activity mediated by exosomes from IFN- $\alpha$-stimulated macrophages (IFN-EXO) was diminished in TIM-1 knockdown HepG2.2.15 cells in comparison to that in cells transfected with control siRNA, as indicated by HBsAg expression (Fig. 1P). In addition, IFN- $\alpha$-induced exosome-mediated antiviral activity only slightly suppressed HBV DNA production in the supernatant of TIM-1 knockdown cells, in contrast to cells transfected with control siRNA (Fig. 1P). Collectively, these findings demonstrated that PtdSer and its receptor TIM-1 act as portals for exosome internalization and the transfer of IFN- $\alpha$-induced antiviral activity against HBV.

Dynamin-2 and cholesterol are required for exosome entry into hepatocytes. The interaction of exosomes with receptors on donor cells can induce the cellular response of internalization through endocytic pathways (29). Endocytosis occurs via several pinocytic mechanisms that include the clathrin-mediated mechanism, macropinocytosis, the caveola-mediated mechanism, and other, less well-defined mechanisms $(30,31)$. The large GTPase dynamin-2 functions at the heart of endocytic vesicle fission in clathrin-mediated endocytosis (CME) and caveola-mediated endocytosis (Fig. 2A) (32). Recent studies showed that dynamin is also responsible for the closure of circular ruffles in macropinocytosis (Fig. 2A) (33). Cholesterol plays essential roles in the formation of caveolae, clathrin-coated pit budding, and membrane ruffling in macropinocytosis (Fig. 2A) (34-36).

To investigate the role of dynamin-2 in exosome entry, we suppressed the function of dynamin-2 in HepG2 cells with the specific inhibitor dynasore. The efficacy of dynasore was confirmed using Alexa Fluor 568-labeled transferrin (Alexa568-TFN), which is the best-characterized cargo protein of CME (Fig. 2B and C). The uptake of PKH26-labeled exosomes was reduced by approximately $60 \%$ following dynasore treatment (Fig. 2C). In addition, the expression of the dominant negative mutant of dynamin-2, Dyn2K44A, also significantly blocked exosome entry (Fig. 2D). We next sought to determine whether cholesterol is necessary for exosome entry into hepatocytes. Using methyl- $\beta$-cyclodextran $(\mathrm{M} \beta C D)$ to extract cholesterol from the plasma membrane of HepG2 cells significantly inhibited PKH26-labeled exosome entry (Fig. 2E and F). The reduction was up to $86 \%$ when treating HepG 2 cells with $10 \mathrm{mM} \mathrm{M} \beta C D$ (Fig. $2 \mathrm{~F}$ ). Masking cholesterol with binding compounds (nystatin and filipin) resulted in milder but still apparent inhibition of exosome uptake by HepG2 cells (Fig. 2E, G, and $\mathrm{H})$. These results indicated that the dynamin-2- and cholesterol-dependent endocytic pathways are required for the entry of exosomes into hepatocytes.

Clathrin- but not caveola-mediated endocytosis is important for exosome uptake and the transmission of IFN- $\alpha$-induced anti-HBV activity. CME, which is the uptake of material into cells from the surface using clathrin-coated vesicles, is the preferred route by which some PtdSer-exposing viruses enter target cells (27). To investigate the dependence of exosome entry on $\mathrm{CME}$, hepatocytes were treated with chlorpromazine (CPZ), an inhibitor of clathrin-coated pit assembly. PKH26-labeled exosome uptake decreased by $34 \%$, and as a positive control, transferrin uptake was inhibited under the same conditions (Fig. 3A and B). Moreover, knockdown of the clathrin heavy chain $(\mathrm{CHC}$ ) also reduced exosome entry into hepatocytes by $34 \%$ (Fig. $3 C$ and D). To further investigate the endocytic pattern engaged in exosome entry, exosomes were stained with PKH67 and administered to HepG2 cells in the presence of Alexa568-TFN. Partial colocalization of exosomes and transferrin was observed $30 \mathrm{~min}$ postinternalization, while little colocalization was captured $1 \mathrm{~h}$ after internalization, indicating rapid clathrin-dependent endocytosis during the early stage of exo- 
A

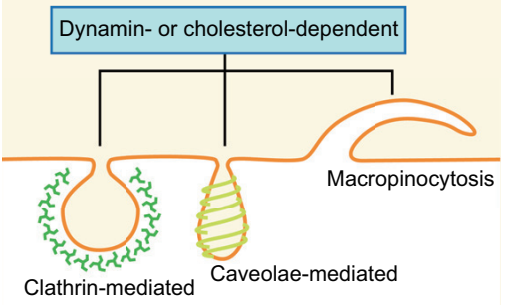

Endocytosis
B

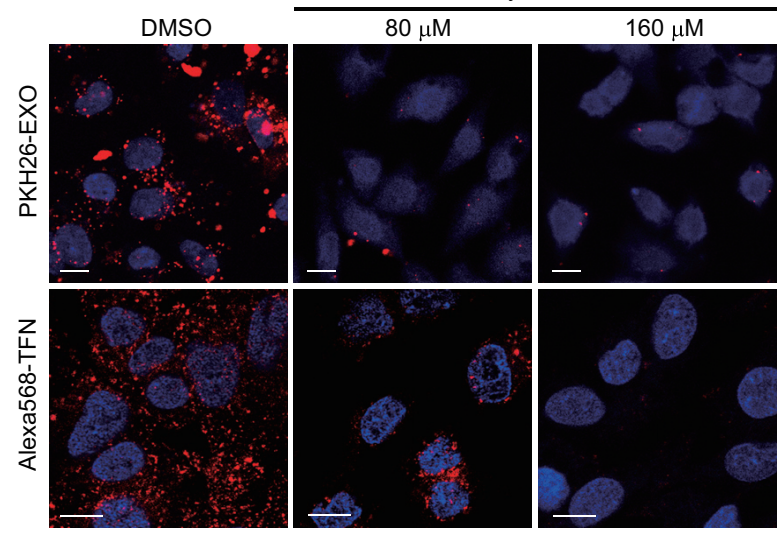

C

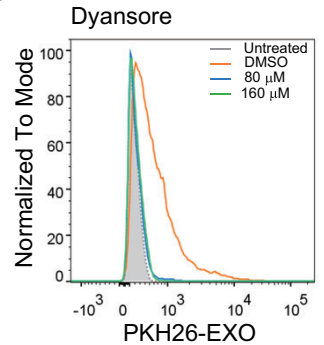

D

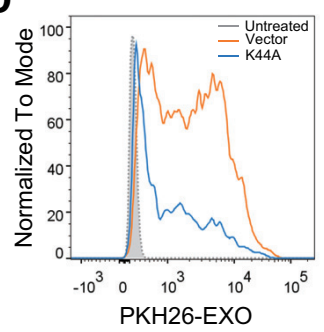

F

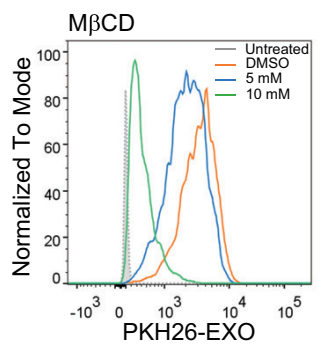

H

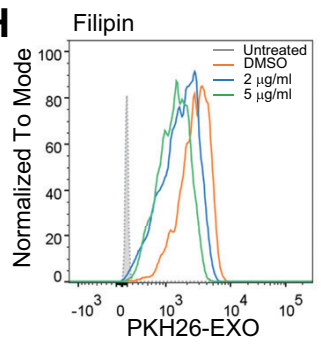

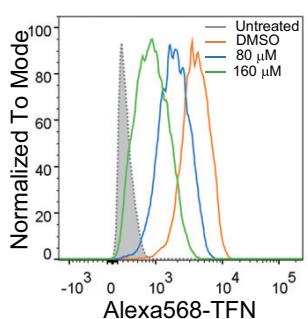

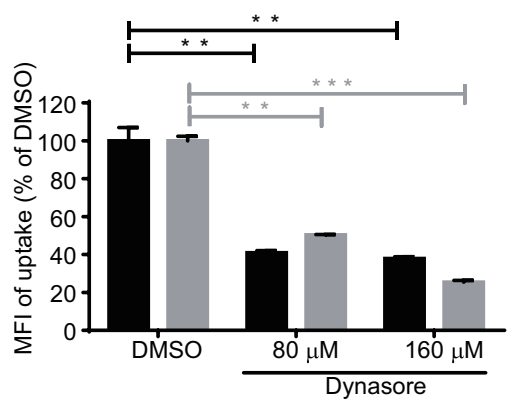

E
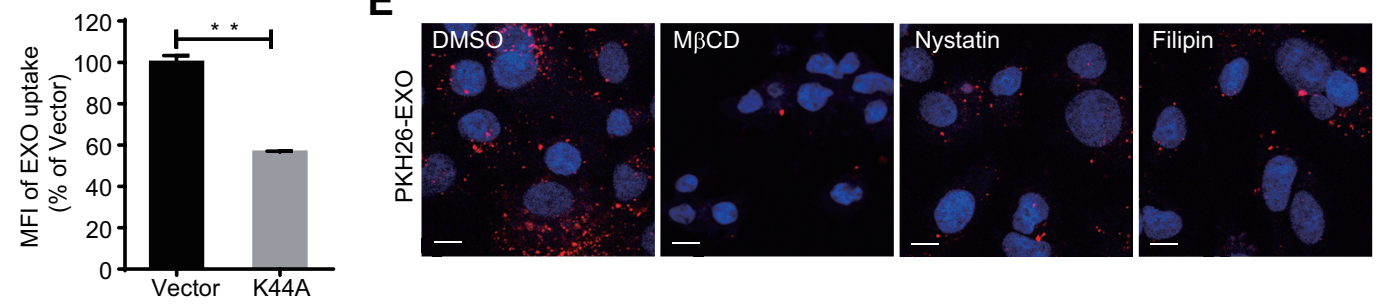

G
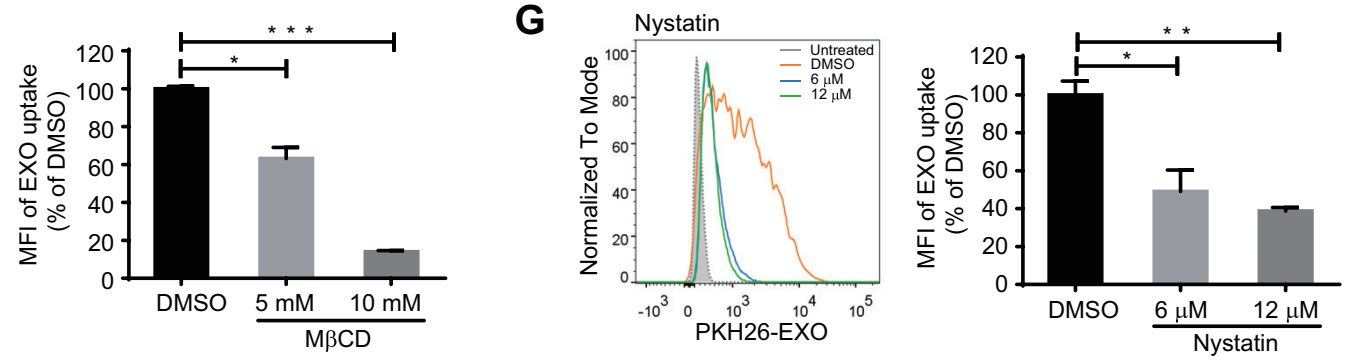

FIG 2 Exosome internalization is dynamin and cholesterol dependent. (A) Schematic representation of the roles of dynamin-2 and cholesterol in various endocytic pathways. ( $B$ and C) Confocal images (B) and flow cytometry analysis (C) of exosome and transferrin internalization by HepG2 cells treated with dynasore. Scale bars: $10 \mu \mathrm{m}$. MFI (right) is normalized to that of dimethyl sulfoxide (DMSO)-treated cells. (D) Flow cytometry analysis of exosome internalization by HepG2 cells transfected with the EGFP-Dyn2K44A mutant. HepG2 cells transfected with the EGFP-tagged dominant negative Dyn2K44A mutant were incubated with PKH26-labeled exosomes. Transfected cells (EGFP+) are gated, and the uptake of exosomes among transfected cells $\left(\mathrm{EGFP}^{+} \mathrm{PKH} 26^{+}\right)$is analyzed

(Continued on next page) 
some internalization (Fig. 3E and F). Scatterplots, Pearson's correlation coefficient (Rr), and an intensity profile were used to quantify the degree of colocalization between PKH67-labeled exosomes and Alexa568-TFN. Partial colocalization between exosomes and transferrin was evidenced by scatterplots, a fraction of which were close to diagonal, and the corresponding Rr was 0.1292 (see Materials and Methods) (Fig. 3E). There were several peak superpositions in the intensity profile (Fig. 3E). Correspondingly, the downregulation of CHC expression in HBV-replicating hepatocytes weakened the IFN- $\alpha$-induced anti-HBV activity transmitted by exosomes in HepG2.2.15 cells, as indicated by viral antigen expression and DNA quantification (Fig. 3G and H).

We then investigated the role of caveola-mediated endocytosis in macrophage exosome entry into hepatocytes. Immunoblotting showed that the expression of endogenous caveolin 1 (CAV1) in HepG2 was low (data not shown), which is consistent with previous reports (37). Moreover, interfering with endogenous caveolin 1 expression did not inhibit the uptake of macrophage exosome by HepG2 (Fig. 3I and J). Thus, caveola-mediated endocytosis may contribute little to exosome uptake by hepatocytes. Together, these data showed that clathrin- but not caveola-mediated endocytosis contribute to exosome uptake and the transfer of IFN- $\alpha$-induced HBV resistance.

Macropinocytosis plays an alternative role in exosome uptake and the transfer of IFN- $\boldsymbol{\alpha}$-induced anti-HBV activity. More than one endocytic route was reported to be used in virus or exosome entry $(29,38)$. Given the incomplete inhibition of exosome entry by blockade of CME and the sustained increase of internalized exosomes in a time-dependent manner (Fig. 3B and D), there might be alternative pathways to support exosome entry into hepatocytes. Macropinocytosis is a fluid-phase type of endocytosis that is accompanied by membrane ruffles regulated by actin rearrangement (33). This process is engaged in apoptotic cell removal and is favored by some viruses that use apoptotic mimicry to enter target cells (27).

The induction of a robust increase in fluid-phase uptake is a hallmark of macropinocytosis (35). The results showed that the uptake of 70-kDa dextran labeled with rhodamine B isothiocyanate (RhoB-dextran), which is a fluid-phase marker specific for macropinocytosis, was enhanced by incubation with macrophage-derived exosomes in HepG2 cells (Fig. 4A). An $\mathrm{Na}^{+} / \mathrm{H}^{+}$exchanger (NHE) is needed for macropinosome formation via the modulation of Rho GTPases at the plasma membrane, and NHE inhibition by 5 -( $N$-ethyl- $N$-isopropyl) amiloride (EIPA) has been widely used as a diagnostic criterion for macropinocytosis (39). The entry of both exosomes and dextran into HepG2 cells was apparently inhibited by EIPA, and a remarkable decrease $(80 \%)$ in exosome uptake was achieved in the presence of $80 \mathrm{nM}$ EIPA (Fig. 4B and C). PAK1 and protein kinase $C$ (PKC) are two serine/threonine kinases that are required for macropinocytosis (35). We found that exosome entry was markedly blocked by the PAK1 inhibitor IPA-3 and the PKC inhibitor rottlerin (Fig. 4D to F). PKC inhibition resulted in a more significant reduction in exosome internalization, by up to $66 \%$ in hepatocytes (Fig. 4F). As a positive control, dextran internalization was greatly inhibited by the two kinase inhibitors (Fig. 4D to F). However, the expression of a dominant negative mutant of Rac1 or Cdc42, two common GTPases that modulate membrane ruffles, had no effect on exosome internalization (Fig. 4G and H), which suggested that macrophage exosomes might enter hepatocytes via a Rac1- or Cdc42-independent route. Next, we reinvestigated the role of macropinocytosis in exosome entry by comparing the distribution patterns of dextran and exosomes after internalization. In contrast to that seen for rapid CME-dependent exosome uptake, confocal images showed consistent colocalization of PKH67-labeled exosomes with RhoB-dextran-filled intracellular vacuoles (Fig. $4 \mathrm{I}$ and J). A highly overlapped distribution was observed $1 \mathrm{~h}$ post-exosome

FIG 2 Legend (Continued)

and presented by histogram graph (left) and MFI (right). MFI is normalized to vector-transfected controls. (E to H) Confocal images (E) and flow cytometry analysis ( $\mathrm{F}$ to $\mathrm{H}$ ) of exosome internalization by HepG2 cells treated with cholesterol inhibitors (M $\beta C D$, nystatin, and filipin). Scale bars: $10 \mu \mathrm{m}$. For flow cytometry analysis, MFIs (right) are normalized to DMSO-treated cells. The error bars indicate the SD. ${ }^{*}, P<0.05 ;{ }^{* *}, P<0.01$; ${ }^{* * *}, P<0.001$ (Student's $t$ test). The data are representative of those from three independent experiments. 
A

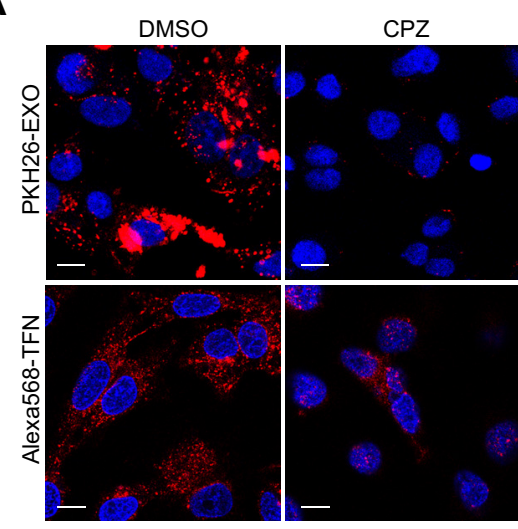

E

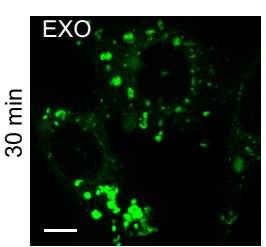

F
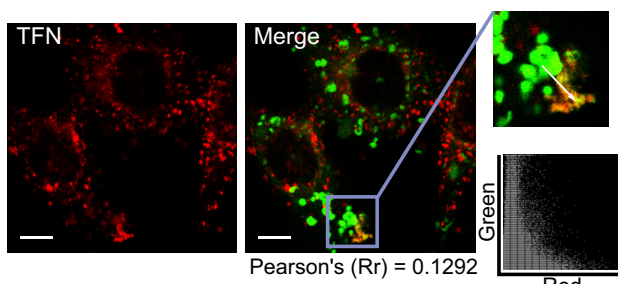

C

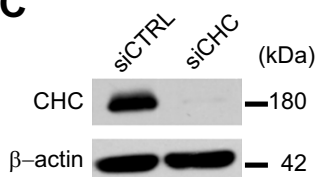

B

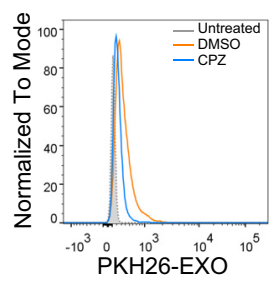

D
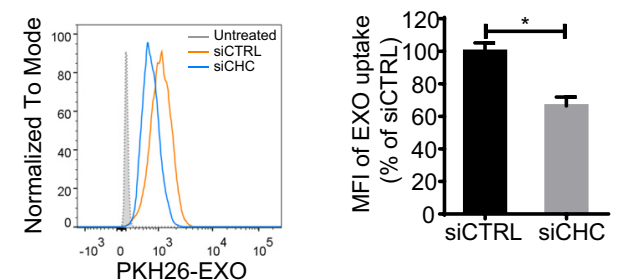
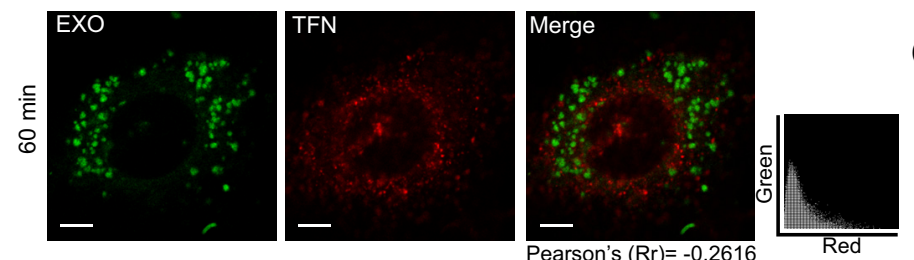

H

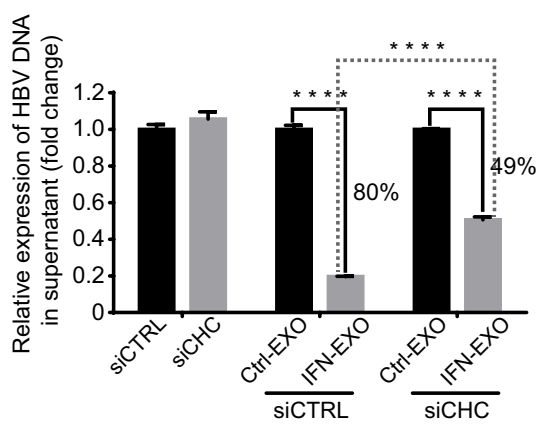

I
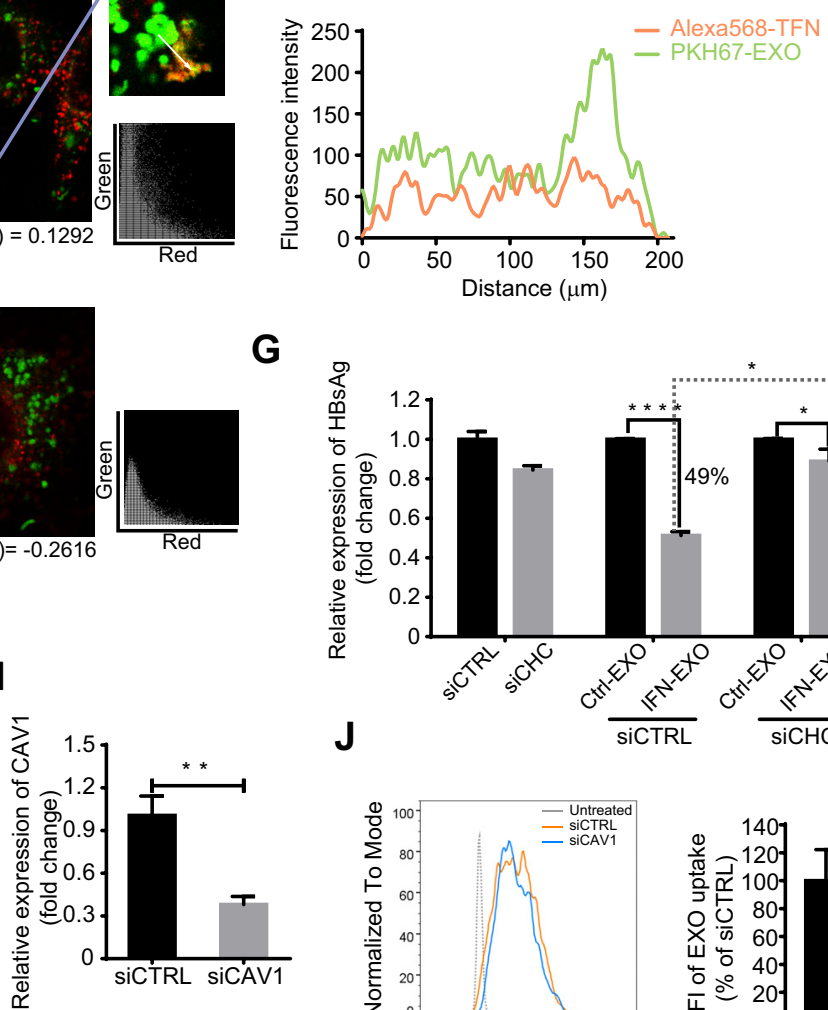

G
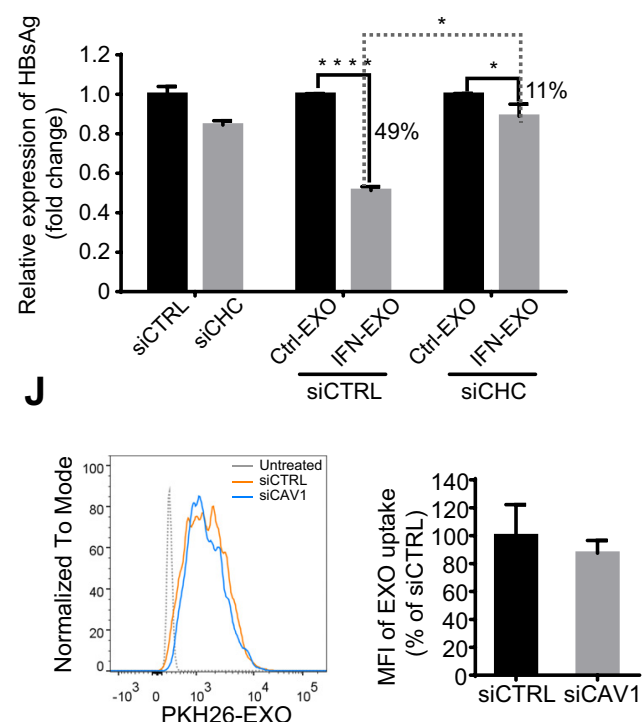

FIG 3 Exosome internalization involves clathrin-mediated endocytosis (CME) and not caveola-mediated endocytosis. (A and B) Confocal images (A) and flow cytometry analysis (B) of exosome and transferrin internalization by HepG2 cells treated with $10 \mu \mathrm{g} / \mathrm{ml}$ of CPZ. Scale bars: $10 \mu \mathrm{m}$. For flow cytometry analysis, MFIs (right) are normalized to that of DMSO-treated cells. (C) Knockdown validation of clathrin heavy chain (CHC) by immunoblotting. (D) Flow cytometry analysis of exosome internalization by HepG2 cells after CHC knockdown. MFls (right) are normalized to that of siCTRL-transfected cells. (E and F) Internalized exosome colocalized with transferrin 30 (E) min and $1 \mathrm{~h}(\mathrm{~F})$ after internalization. The cells were fixed and analyzed by confocal microscopy. Scatterplots and Pearson's correlation coefficients for the overlap of red (Alexa568-transferrins) and green (PKH67-labeled exosomes) pixel intensities corresponding to the images are presented. Intensity profiles are used to describe the distribution along the indicated white arrow in the region of interest (ROI). Scale bars: $5 \mu \mathrm{m}$. (G and H) Blockade of IFN- $\alpha$-induced anti-HBV activity transmission by CHC knockdown. HepG2.2.15 cells transfected with either siCHC or siCTRL were treated with IFN-EXO or Ctrl-EXO. HBsAg and HBV DNA levels in the medium were measured by ELISA (G) or quantified by qPCR (H). (I) Knockdown validation of caveolin 1 (CAV1) at the mRNA level by qPCR. (J) Flow cytometry analysis of exosome internalization by HepG2 cells with CAV1 knocked down. MFI (right) is normalized to that of siCTRL-transfected cells. The error bars indicate the SD. ${ }^{*}, P<0.05 ;{ }^{* *}, P<0.01 ;{ }^{* * *}, P<0.0001$ (Student's $t$ test). The data are representative of those from three independent experiments. 
A

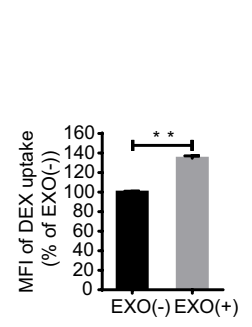

B

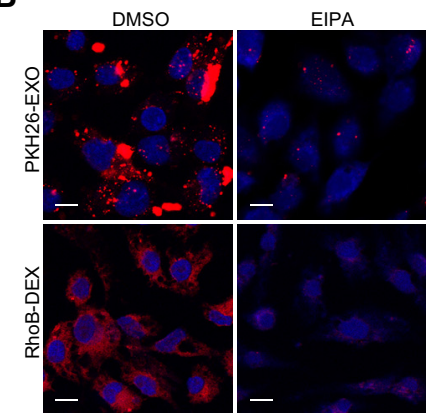

D

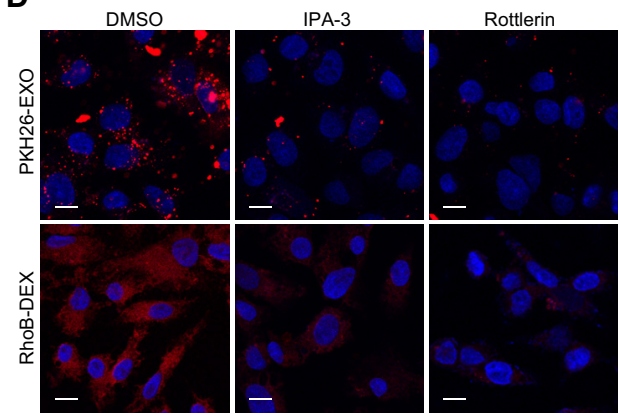

C
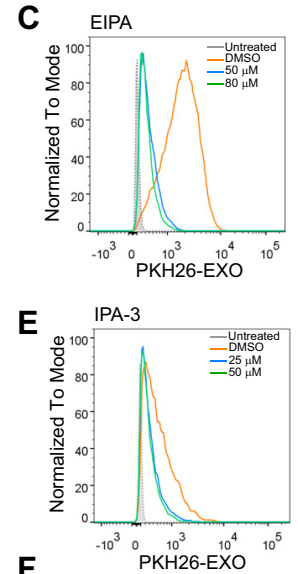

$\mathbf{F}$

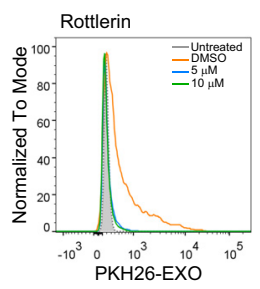

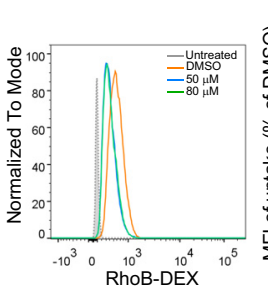
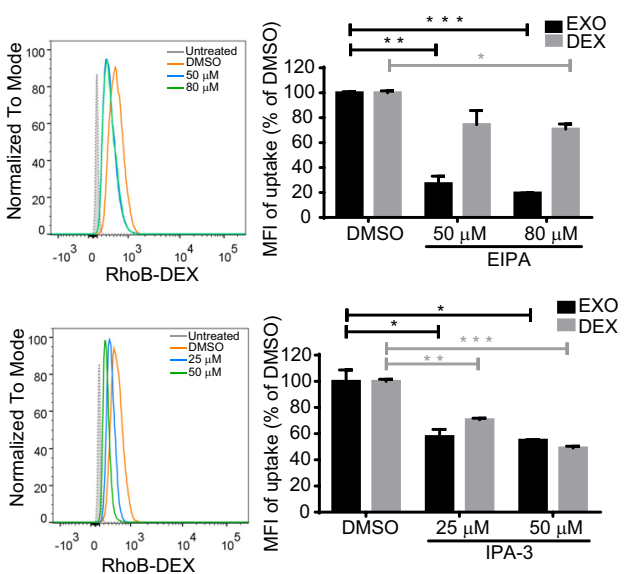
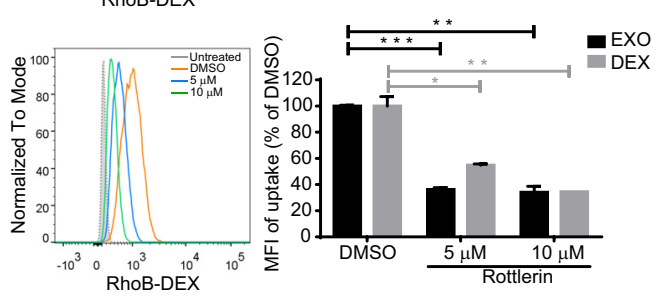

G
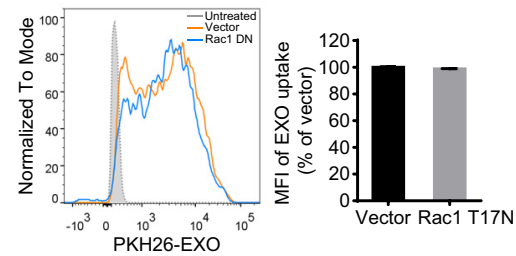

H

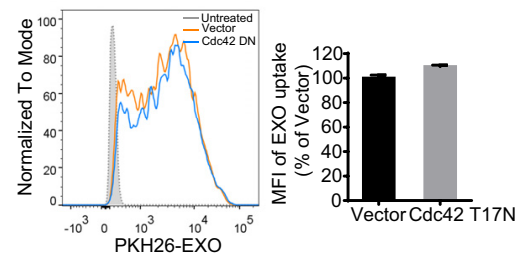

I
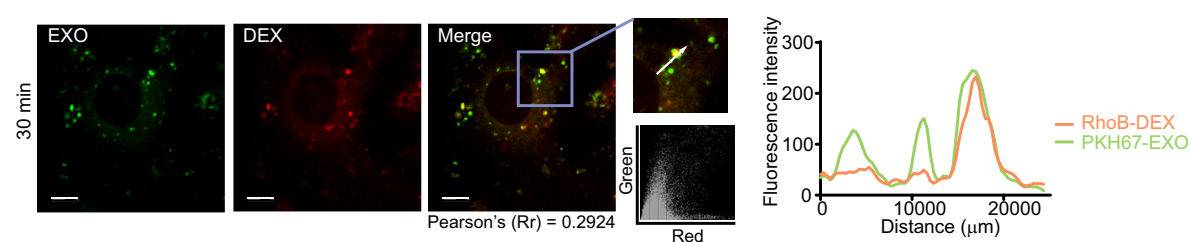

$\mathbf{J}$
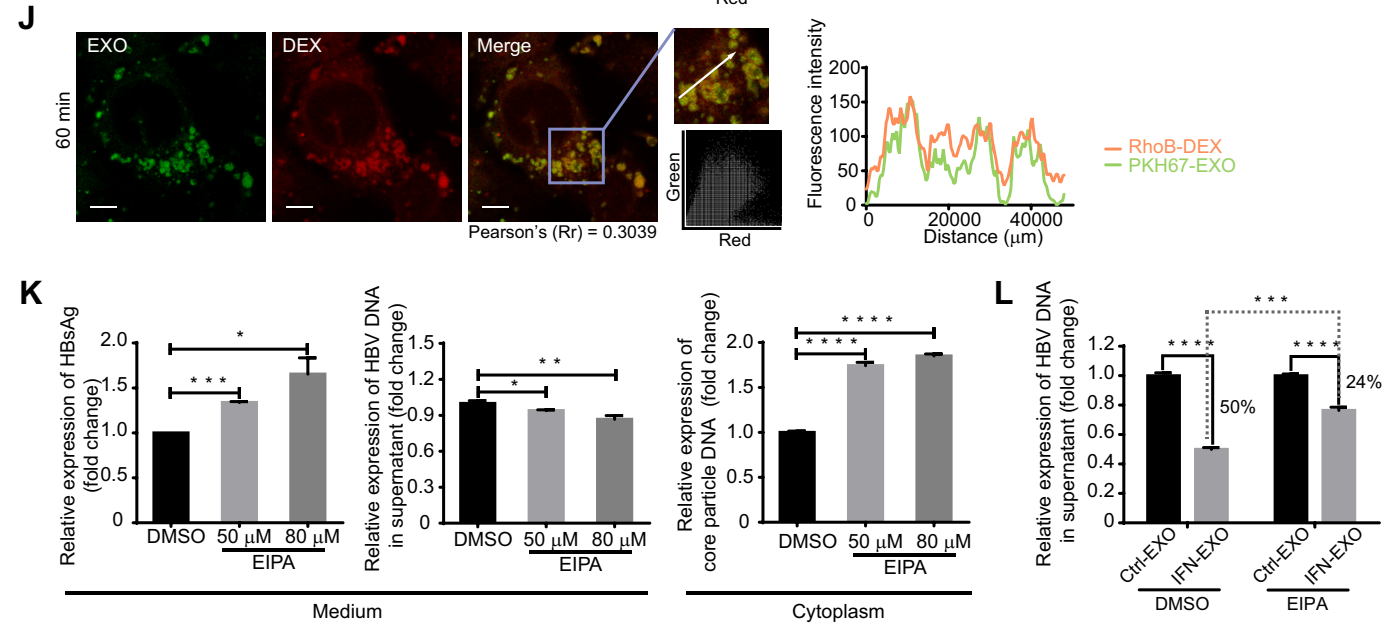

FIG 4 Exosome internalization involves macropinocytosis. (A) Preincubation with exosomes increased dextran uptake in HepG2 cells RhoB-dextran (RhoB-DEX) uptake by HepG2 cells pretreated with exosomes [EXO(+)] was analyzed by flow cytometry, and the MFI is normalized to that of untreated cells $[\mathrm{EXO}(-)]$. (B and C) Confocal images (B) and flow cytometry analysis (C) of exosome and 
internalization and was confirmed by the corresponding scatterplots, colocalization coefficient, and intensity profile (Fig. 4J).

We then further investigated the role of macropinocytosis in exosome-mediated HBV resistance transfer in HepG2.2.15 with macropinocytosis inhibited by EIPA. Unexpectedly, after treatment with EIPA, the amounts of secreted HBsAg and intracellular core-particle-associated DNA increased, and HBV DNA in the culture medium slightly decreased (Fig. 4K). Even though EIPA treatment seemed to induce pleiotropic effects in dose-dependent manner, results still indicated that the inhibition of macropinocytosis in HepG2.2.15 cells partially blocked the IFN- $\alpha$-induced anti-HBV activity mediated by exosomes derived from IFN-treated macrophages (Fig. 4L).

Thus, we concluded that macropinocytosis served as a sustained alternative route that was active from the early stage of exosome internalization and cooperated with CME to ensure hepatocytes access to exosome-mediated HBV resistance.

Exosomes expose cargo through membrane fusion in late endosomes/multivesicular bodies. Once internalized within primary endocytic vesicles, the incoming substances traffic into the endosomal system (38). The endocytosed substances are routed from early endosomes (EEs) to late endosomes (LEs, often taking the form of MVBs) and lysosomes for degradation (38). Membrane fusion-induced endosome penetration is commonly manipulated by viruses or delivery vectors to send viral genomes or biologics to the cytosol before lysosomal degradation (40-43). It remains unknown whether a similar membrane fusion strategy is adopted for exosomal cargo release in endosomes after internalization (Fig. 5A).

We first used time-lapse microscopy to track membrane fusion events in live hepatocytes incubated with macrophage-derived exosomes prelabeled with selfquenching amounts of the hydrophobic dye rhodamine C18 (R18). R18 is commonly used as a fluorescent probe to detect virus-induced membrane fusion. The probe is incorporated into membranes at high concentrations to cause self-quenching, and dequenching of the probe occurs when membrane fusion decreases in density $(40,44)$. The dequenching signal of membrane fusion was first captured approximately $45 \mathrm{~min}$ after treating HepG2 cells with R18-labeled exosomes, and fusion events followed within $1 \mathrm{~h}$ (Fig. 5B). The fluorescence intensity profile showed persistent enhanced R18 fluorescence for the fusion spots (Fig. 5C).

EEs and LEs/MVBs are major fusion sites for some viruses to deliver nucleocapsids and release nucleocapsids to the cytosol (45). To locate the exact site at which membrane fusion occurred after exosome internalization, we performed colocalization experiments using a variety of endosomal markers. Endosomal compartments in HepG2 cells were labeled via transient transfection of plasmids encoding cyan fluorescent protein (CFP)-fused markers for EEs (RAB5), LEs/MVBs (RAB7), and intraluminal vesicles (ILVs) in MVBs (CD63). The dequenching signal of membrane fusion was colocalized with the LE marker CFP-RAB7 and the ILV marker CFP-CD63 in live HepG2 cells after treatment with R18-labeled exosomes, while no colocalization was observed with markers for EEs (CFP-RAB5) (Fig. 5D). Hence, LEs/MVBs might be the proper site for the membrane fusion of macrophage-derived exosomes after exosome internalization.

FIG 4 Legend (Continued)

dextran internalization by HepG2 cells treated with EIPA. Scale bars: $10 \mu \mathrm{m}$. For flow cytometry analysis, MFIs (right) are normalized to that of DMSO-treated cells. (D to F) Confocal images (D) and flow cytometry analysis (E and F) of exosome and dextran internalization by HepG2 cells treated with IPA-3 or rottlerin. Scale bars: $10 \mu \mathrm{m}$. For flow cytometry analysis, MFIs (right) are normalized to that of DMSO-treated cells. (G and H) Exosome uptake is independent of Rac1 or Cdc42. Flow cytometry analysis of exosome internalization by HepG2 cells transfected with EGFP-Rac1 dominant negative mutant (G) or EGFP-Cdc42 dominant negative mutant $(\mathrm{H})$, followed by incubation with PKH26-labeled exosomes. Transfected cells (EGFP+) are gated, and the uptake of exosomes among transfected cells (EGFP+ $\mathrm{PKH}^{+} 6^{+}$) was analyzed as described above. (I and J) Internalized exosome colocalized with dextran $30 \mathrm{~min}(\mathrm{I})$ and $1 \mathrm{~h}(\mathrm{~J})$ after internalization. The colocalization of Rho-dextran (red) with PKH67-labeled exosomes (green) was analyzed as described above. Scale bars: $5 \mu \mathrm{m}$. (K) HepG2.2.15 cells were treated with DMSO or EIPA. The level of HBsAg in the culture medium (supernatant) was detected by ELISA. HBV DNA in the culture medium and intracellular core particle DNA were quantified by qPCR. (L) Blockade of IFN- $\alpha$-induced anti-HBV activity transmission by EIPA treatment. HepG2.2.15 cells were pretreated with DMSO or EIPA, and the drugs were present continuously during following incubation with IFN-EXO or Ctrl-EXO. HBV DNA levels in the medium were quantified by qPCR. The error bars indicate the SD. ${ }^{*}, P<0.05 ;{ }^{* *}, P<0.01 ;{ }^{* * *}, P<0.001 ;{ }^{* * *}, P<0.0001$ (Student's $t$ test). The data are representative of those from three independent experiments. 
A

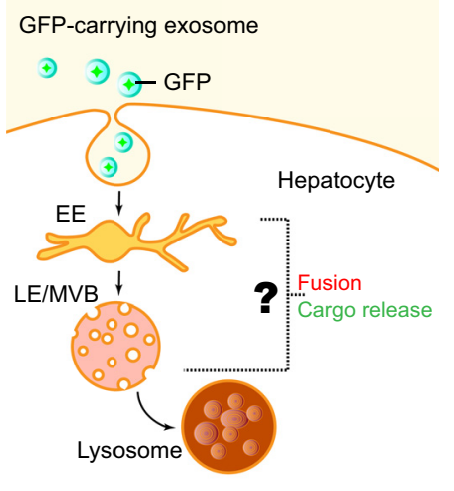

C

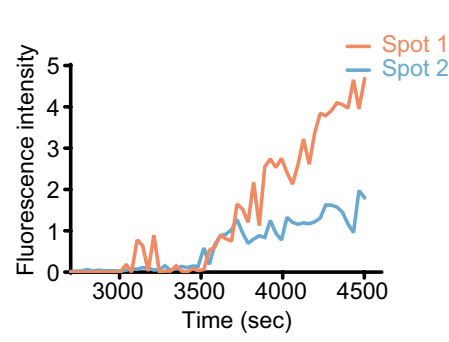

E R18-labeled GFP-EXO

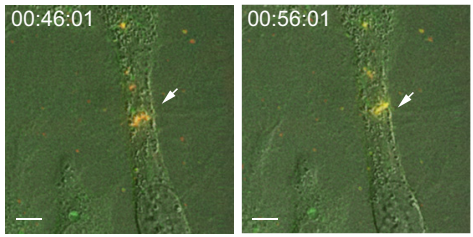

F

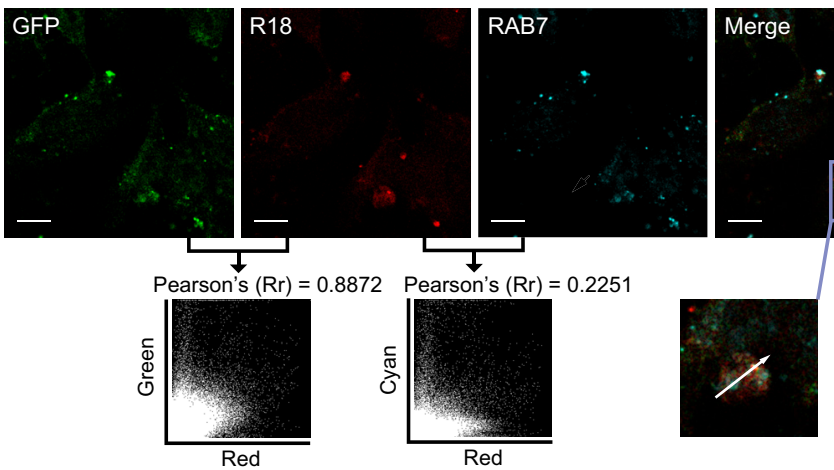

R18-EXO
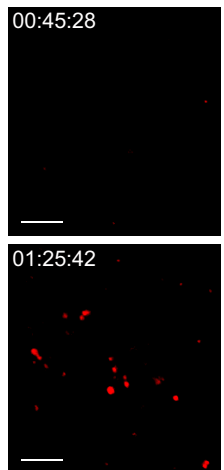

D R18-EXO RAB5

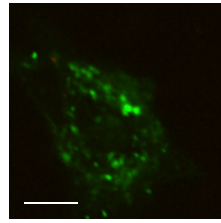

Red
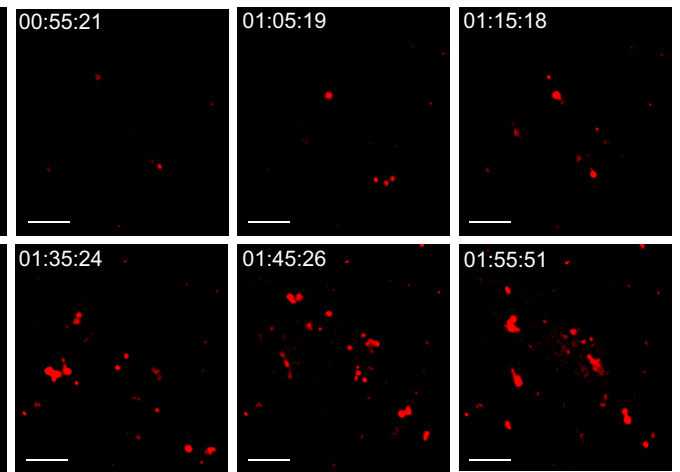

R18-EXO RAB7

R18-EXO CD63
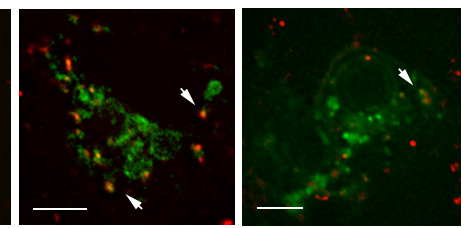

Pearson's $(\mathrm{Rr})=0$ Pearson's $(\mathrm{Rr})=0.2622$ Pearson's $(\mathrm{Rr})=0.2497$

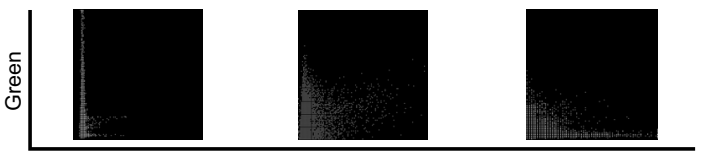

Red
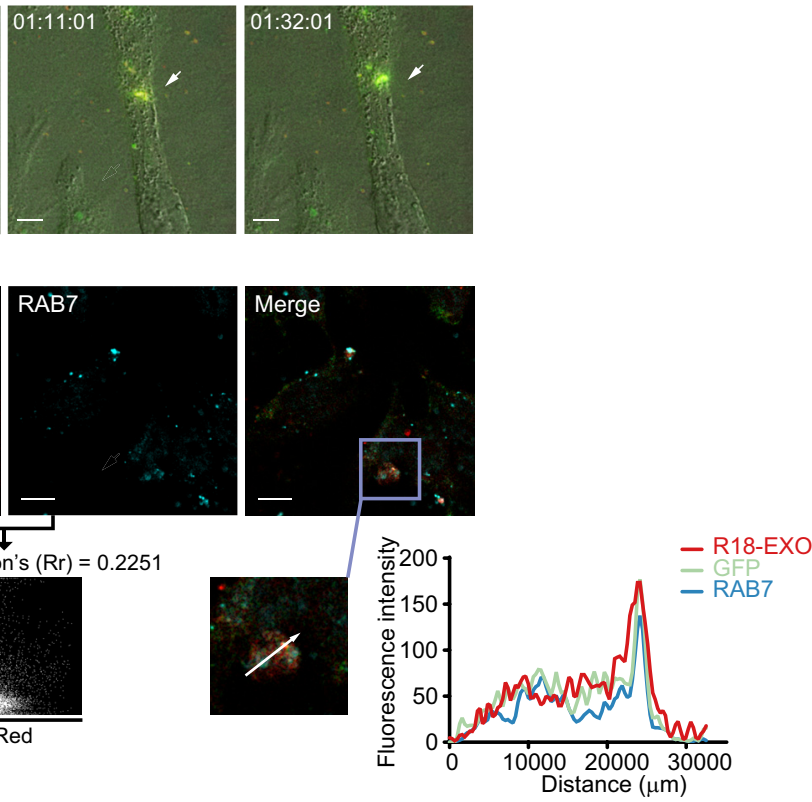

FIG 5 Membrane fusion of GFP-carrying exosomes occurs in LEs/MVBs. (A) Hypothetical model of exosome fusion and cargo release in endosomes. (B) Images of R18 dequenching triggered by exosome membrane fusion. R18-dequenching fusion spots (red) were tracked and imaged at the indicated time points via time-lapse microscopy. Scale bars: $5 \mu$ m. (C) Time-intensity profiles of R18 fluorescence of two representative dequenching spots in panel B. (D) Membrane fusion signals of exosomes colocalized with the LE marker CFP-RAB7 and the ILV marker CFP-CD63. Dynamic colocalization events of dequenching signals (red) with cellular markers (CFP pseudocolored green) were tracked via time-lapse microscopy. Scatterplots and Pearson's correlation coefficients for colocalization are presented below the images. Scale bars: $5 \mu \mathrm{m}$. (E) Color shift induced by ongoing fusion process of GFP-carrying exosomes prelabeled with self-quenching concentrations of R18 was observed and imaged via time-lapse microscopy. Scale bars: $5 \mu \mathrm{m}$. (F) Membrane fusion signals of GFP-carrying exosomes colocalized with the LE marker RAB7. Scatterplots and Pearson's correlation coefficient between the signals of GFP and R18 or the signals of R18 and RAB7 are presented. Fluorescence intensity profiles of GFP, RAB7, and R18 along the indicated white arrow in the ROI are also presented. Scale bars: $10 \mu \mathrm{m}$. 
To track exosomal cargo after membrane fusion, the live dynamics of exosomal cargo in hepatocytes were tested by monitoring the membrane fusion events of R18-labeled GFP-carrying exosomes using time-lapse microscopy. At the beginning of the experiment, orange fluorescence was observed at the fusion site due to the combined fluorescence emitted by dequenching R18 inserted into exosome membranes and GFP encapsulated in exosomes. As fusion proceeded, the extreme dilution of the R18-labeled membrane components increased the fluorescence of the gradually exposed GFP. A complete color switch was accomplished when the exosomal cargo GFP was totally uncoated and "released" (Fig. 5E). In addition, confocal images proved again that LEs were the site of membrane fusion for GFP-carrying exosomes (Fig. 5F). The colocalization coefficient of R18 and GFP was approximately 0.9 in HepG2 cells, indicating a high frequency of fusion events among internalized exosomes. Together, these data indicated that LEs/MVBs provided the proper conditions for exosome fusion and cargo uncoating, which might promote exosomal cargo release based on endosome penetration.

LBPA contributes to exosome fusion and the uncoating of exosomal cargo. Anionic lipids are beneficial for endosome penetration (45). A high concentration of anionic lipids makes LEs a suitable location for endosome leakage via membrane fusion. Notably, the LE-specific anionic lipid lysobisphosphatidic acid (LBPA) assists both virus and delivery vectors in achieving efficient cytosolic access via membrane fusioninduced endosome penetration (41-43, 45-47).

The accumulation of PKH26-labeled exosomes in the LBPA-rich structure suggested a potential interaction between the two components (Fig. 6A). Partial colocalization between the dequenching R18 of exosomes and LBPA signals indicated the participation of LBPA in the membrane fusion of exosomes in LEs/MVBs (Fig. 6B). To verify the dependence of exosome fusion on LBPA, HepG2 cells were preincubated with an anti-LBPA blocking antibody $(40,41)$ and the dequenching signals of R18-labeled exosomes were tracked via time-lapse microscopy. Pretreatment with an anti-LBPA blocking antibody produced significant inhibition of membrane fusion, as suggested by the decayed R18 dequenching of exosomes (Fig. 6C). The fluorescence intensity profile of tracked fluorescent puncta further manifested the dependence of exosome fusion on LBPA (Fig. 6D). To investigate the contribution of LBPA to the potential intracellular release of exosomal cargo before lysosomal degradation, we incubated LBPA-blocked HepG2 cells with GFP-carrying exosomes and judged the delivery efficiency of exosomal cargo to lysosomes based on the colocalization efficiency between GFP and lysosomes. The incidence of colocalization increased significantly in cells pretreated with the anti-LBPA blocking antibody, as indicated by the 2-fold increase in the colocalization coefficient (0.5487) in comparison to control cells (Fig. 6E). This finding suggested that some exosomal cargo might escape from endosomes to avoid lysosomal degradation via LBPA-dependent membrane fusion in LEs/MVBs. Surprisingly, blocking LBPA intensively increased intracellular core-particle-associated DNA and HBV DNA in the culture medium of HepG2.2.15 cells, while the level of HBsAg significantly decreased (Fig. 6F). This outcome impeded further investigations of LBPA in exosomemediated antiviral activity transmission. Nevertheless, combining results described above, we came to the conclusion that LBPA is very important for exosome fusion and the uncoating of exosomal cargo.

\section{DISCUSSION}

In this report, we demonstrate that macrophage-derived exosomes utilize virus entry machinery and pathway to proffer IFN- $\alpha$-induced HBV resistance to hepatocytes. We have presented evidence that macrophage exosomes engage TIM-1, a PtdSer receptor, to enter hepatocytes and undergo rapid CME or sustained macropinocytosis. Our data also suggest that LEs/MVBs are the primary location for LBPA-mediated exosome fusion and accompanying exosomal cargo uncoating for potential intracellular release. The endocytic pathway and membrane fusion in endosomes provide an ideal strategy for exosomes from IFN- $\alpha$-induced macrophages to deliver antiviral 
A

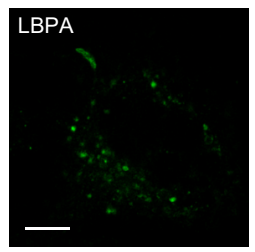

B

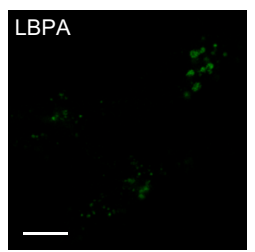

C

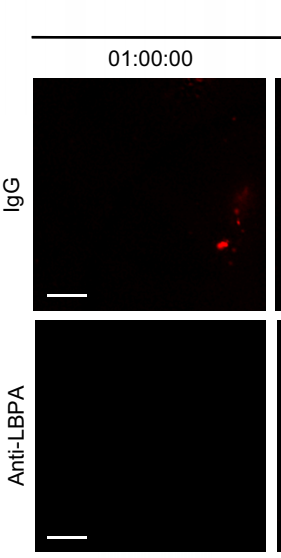

E
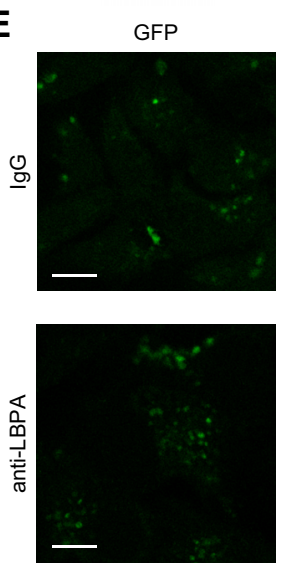
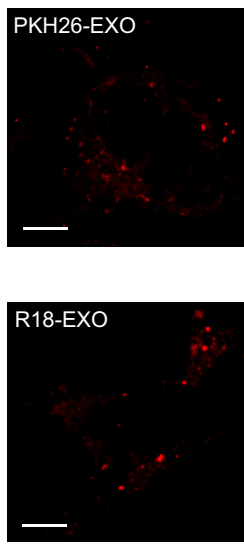

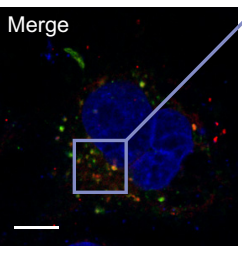

Pearson's $(\mathrm{Rr})=0.1329$

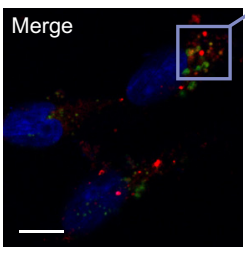

Pearson's $(\mathrm{Rr})=0.0714$
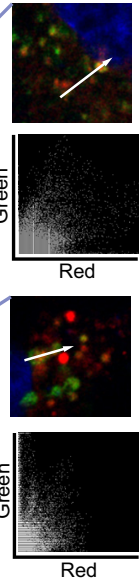

Red
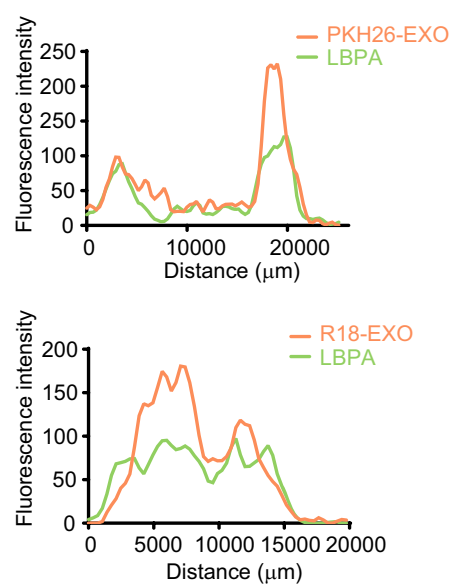

D $\quad-\operatorname{Spot} 1(\mathrm{lgG})$
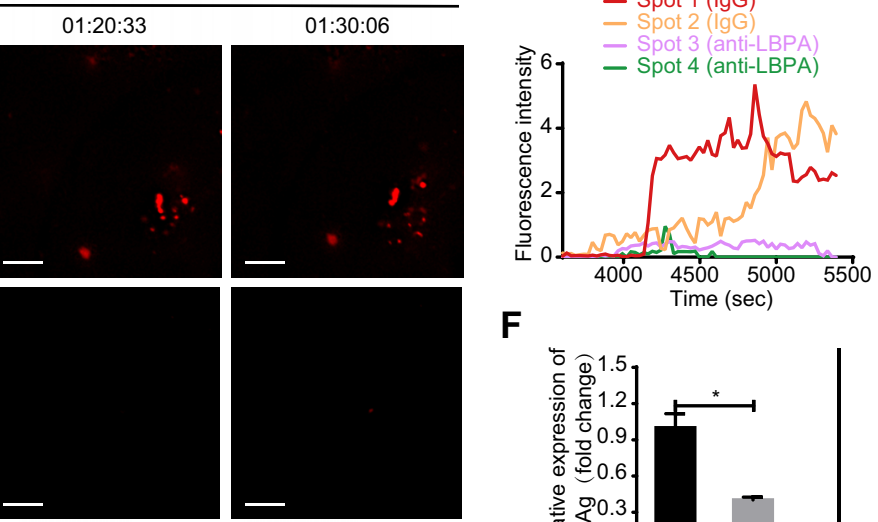

F
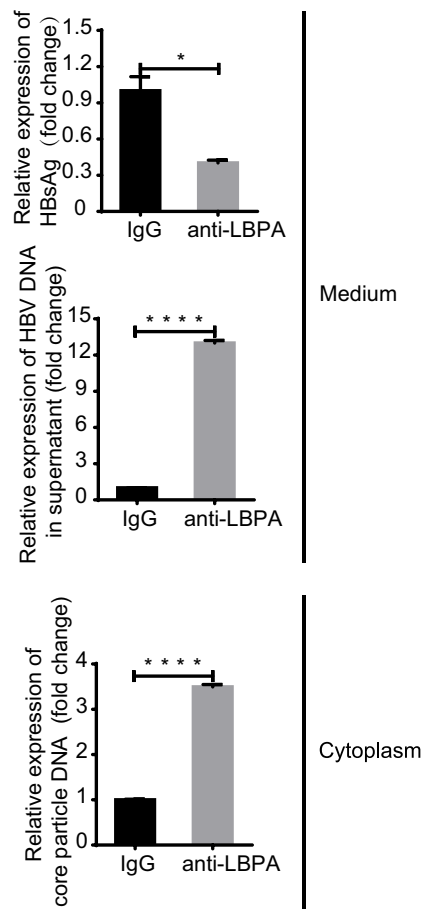

FIG 6 LBPA is required for exosome fusion and cargo uncoating. (A) Accumulation of PKH26-labeled exosomes in LBPA-rich vacuoles. Colocalization of PKH26 (red) with LBPA (green) was analyzed as described in the text. Scale bar: $10 \mu \mathrm{m}$. (B) Membrane fusion signals of dequenching R18-exosomes colocalized with LBPA. Colocalization of dequenching signals (red) with LBPA (green) was analyzed as described in the text. Scale bars: $10 \mu \mathrm{m}$. (C) Inhibition of exosome fusion by antibodies against LBPA. Fusion spots of dequenching R18-exosomes in HepG2 cells pretreated with $50 \mu \mathrm{g} / \mathrm{ml}$ of anti-LBPA or anti-lgG overnight were tracked and photographed at the indicated time points. Scale bars: $5 \mu \mathrm{m}$. (D) Time-intensity profiles of R18 fluorescence of four representative dequenching spots in panel C. (E) Increase in colocalization of the exosomal cargo GFP with lysosomes after exposure to antibodies against LBPA. HepG2 cells pretreated with anti-LBPA or anti-IgG overnight were incubated with GFP-carrying exosomes in the presence of LysoTracker. Colocalization of GFP (green) with lysosomes (red) was analyzed via scatterplots and Pearson's correlation coefficients. Scale bars: $10 \mu \mathrm{m}$. (F) HepG2.2.15 cells were pretreated with anti-lgG or anti-LBPA overnight. The level of HBsAg in the culture medium (supernatant) was detected by ELISA. HBV DNA in the culture medium and intracellular core particle DNA were quantified by qPCR. The error bars indicate the SD. ${ }^{*}, P<0.05 ;{ }^{* * * *}, P<0.0001$ (Student's $t$ test). The data are representative of those from three independent experiments. 


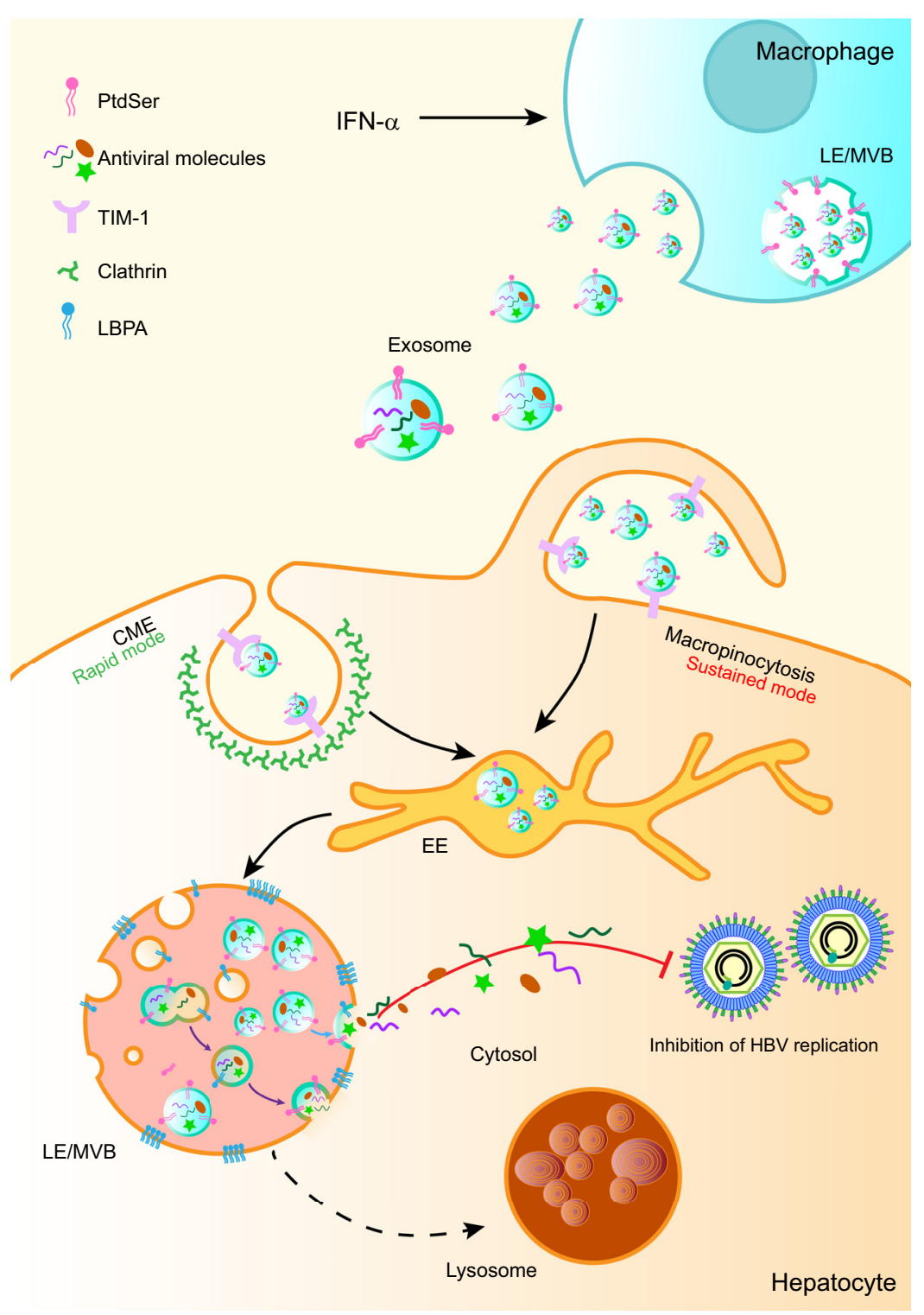

FIG 7 Proposed model of exosome entry and delivery of IFN- $\alpha$-induced HBV resistance. After binding to TIM-1, exosomes from IFN- $\alpha$-stimulated macrophages enter HBV-replicating hepatocytes through CME (rapid mode) and macropinocytosis (sustained mode). Endocytosed exosomes traffic to LEs/MVBs and fuse with LBPA-rich ILVs. Trapped antiviral cargo in the ILVs is released to the cytosol via the back-fusion of ILVs with the limiting membrane of LEs/MVBs (violet arrow). Alternatively, ILV-derived exosomes release antiviral cargo via direct fusion with LEs/MVBs (blue arrow).

activity and control HBV replication in hepatocytes (Fig. 7). Interestingly, we also found that inhibition of exosome entry machinery and pathway may disturb HBV or subviral particle biogenesis.

Exosomes have been shown to interact with membrane receptors on target cells to facilitate subsequent endocytosis (29). Recently, a virus endocytic modelapoptotic mimicry-was suggested to play a role in exosome entry $(10,27)$. As former ILVs form by inward budding of the LE/MVB-limiting membrane, exosomes are thought to expose PtdSer, an apoptotic marker, on the external leaflet of the membrane and initiate PtdSer receptor-engaged uptake (48). Apoptotic mimicry has been used by hepatotropic hepatitis A virus (HAV) for infection, in which the virus is cloaked in a PtdSer-containing envelope by hijacking the exosome secretion pathway and entering target cells via TIM-1-mediated internalization (27, 49, 50). Although PtdSer is generally expressed on the external membrane of exosomes 
from different cell types, TIM-1 uniquely mediated the entry of macrophage-derived exosomes into hepatocytes. The result suggested that macrophage exosomes might exploit an endocytic strategy similar to apoptotic mimicry as HAV uses to enter cells. However, it remains to be explained why TIM-1-mediated uptake is not applicable to exosomes from other cell types, such as HepG2 and LX-2. Do nonmacrophage-derived exosomes choose other PtdSer receptors to enter hepatocytes? Otherwise, do molecules other than PtdSer on the surface of these exosomes permit the entry into hepatocytes? Several adhesion molecules, including integrins, immunoglobulins, and proteoglycans, were reported to be involved in exosome attachment to cells $(10,29)$. The possibility cannot be excluded that these molecules may also facilitate exosome binding to hepatocytes. We also found that exosomes from cells of nonhepatic tissues hardly entered hepatocytes, which implied a role for tissue specificity in exosome internalization. The incapability of entering hepatocytes might be due to lack of particular molecules on these exosome surfaces. A previous study showed that MUC1-positive milk-derived exosome could enter monocyte-derived dendritic cells via lectin, while MUC1-negative exosomes fail to be internalized by DCs (51).

Macrophage exosomes seem to be welcomed by multiple recipient cells, including hepatic cells (HepG2 and LX-2) and nonhepatic cells (MGC-803). However, other mediators, except TIM-1, appear to be responsible for macrophage exosome entry into LX-2 and MGC-803 cells. It may be the high expression of TIM-1 that makes TIM-1medaited uptake the preferential route for macrophage exosomes to enter hepatocytes. Cell type specificities of exosome internalization have been reported in cancer research. Exosomes from mantle cell lymphoma $(\mathrm{MCL})$ were rapidly and preferentially internalized by $B$ lymphocytes, and minor fractions were taken up by $\mathrm{T}$ cell leukemia and bone marrow stroma cell lines (52). Another example is that CD11 b leukocytes took up pancreatic-adenocarcinoma-derived exosomes more preferentially than $\mathrm{T}$ and $B$ cells (53). In general, the hepatocyte receptors for uptake of exosomes from different cell types are not the same, and TIM-1-mediated uptake in HepG2 cells is somehow specific to macrophage exosomes.

Adhesion to receptors commonly results in a cellular response of internalization through endocytic pathways (38). The type of stimulated endocytic pathway is related to endocytosed particle size and recipient cells (54-56). Immune cells, including phagocytes (DCs and macrophages) and "nonprofessional" phagocytes ( $T$ cells), can take up exosomes through phagocytosis or macropinocytosis $(55,56)$. Exosome internalization seems more complicated in nonphagocytes, in which clathrin-mediated and caveola-mediated endocytosis, macropinocytosis, and some nonclassic lipid raft-dependent endocytosis are involved (23, 24, 26, 29). Some studies even showed that exosomes from tumor cells could fuse directly with target cells (57). It is believed that various combinations of endocytic mechanisms are responsible for exosome entry in different cell types (29). PtdSer exposure is exploited by some viruses as an apoptotic disguise which triggers subsequent CME or macropinocytosis for virus entry (27).

Correspondingly, we found that TIM-1-mediated macrophage exosome entry also depended on rapid CME and sustained macropinocytosis. However, Rac1 and Cdc42, two Rho GTPases that are usually engaged in macropinocytosis, do not appear to be involved in macrophage exosome uptake by hepatocytes. This finding is inconsistent with the interference of exosome entry by EIPA, which inhibits the activation of Rac1 and $\mathrm{Cdc4}$ by altering the submembranous $\mathrm{pH}$ (39). Therefore, exosome entry into hepatocytes may rely on undefined EIPA-sensitive Rho GTPases. Moreover, Rac1- and Cdc42-independent macropinocytosis is reportedly invoked during influenza A virus (IAV) entry (58). Related studies also showed that circular ruffling and macropinocytosis independent of Rac1 or Cdc42 could be triggered by the nonreceptor tyrosine kinase c-src (59).

Little research to date has focused on the fates of exosomes and exosomal cargo after internalization (10). Endocytosed substances are usually directed to the endo- 
somal system composed of EEs, recycling endosomes (REs), LEs, and lysosomes, where they are sorted, processed, recycled, stored, and degraded (38). LEs often take the form of MVBs where ILVs, the precursor of exosomes, are born (60). Viruses and delivery vectors exploit endosomes for penetration into the cytosol through membrane fusion to deliver viral genomes or biologics $(42,43,45)$. Using a live-cell imaging system and a fusion probe (R18), we found that LEs/MVBs were also the potential site of exosome fusion initiation, followed by cargo uncoating. Notably, the persistence of R18 dequenching signals for several minutes indicated that exosome fusion was trapped in an endosomal subcompartment, identical to the colocalization of fusion signals with an ILV marker (CD63) (Fig. 5C and D) (40).

Previous studies have shown that a high concentration of anionic lipids in LEs provides an appropriate environment for endosome penetration $(45,61)$. It was reported that the presence of anionic lipids in the target membrane promoted membrane fusion efficiency for some enveloped viruses $(41,46,62)$. LBPA is a specific anionic lipid in LEs and is thought to promote ILV budding and back-fusion $(60,63)$ during MVB biogenesis. Research has suggested that vesicular stomatitis virus (VSV) loads nucleocapsids into ILVs through membrane fusion and penetrates LEs/MVBs through LBPAdependent back-fusion between the ILV membrane and the endosome-limiting membrane (41). In addition, LBPA is also required for efficient cytosolic access of delivery vectors, including dfTAT and phosphorothioate-modified antisense oligonucleotides (PS-ASO) $(42,43)$. Our results showed that the fusion sites of exosomes were colocalized with LBPA. Moreover, LBPA antibodies inhibited the membrane fusion of endocytosed exosomes and accelerated the transport of exosomal cargo to lysosomes. It is possible that some exosomal cargo may avoid lysosomal degradation via LBPA-dependent membrane fusion in LEs/MVBs. Given the above-described results, we hypothesize that LBPA facilitated the fusion of exosomes from IFN- $\alpha$-stimulated macrophages with ILVs in LEs/MVBs and that exosomal antiviral cargo are then reloaded into fused ILVs and released after back-fusion with the limiting membrane of LEs/MVBs. As former ILVs formed in LEs/MVBs, endocytosed exosomes with ILV properties may also be qualified for direct fusion with the limiting membrane of LEs/MVBs to release cargo.

The inhibition of entry route of macrophage exosomes from TIM-1 to endocytic pathways (CME and macropinocytosis) significantly attenuated exosome-mediated IFN- $\alpha$-induced anti-HBV transmission. However, unexpected impacts on HBV replication and viral or subviral particle secretion were observed after manipulating related entry pathways. TIM family proteins were reported to inhibit the release of multiple viruses, including HIV-1, murine leukemia virus (MLV), and Ebola virus (EBOV) (64). The phosphatidylserine-binding capability of TIM-1 is responsible for the inhibition of HIV release (64). Considering the decrease of HBV DNA in the culture medium after TIM-1 knockdown, TIM-1 seems to be related to HBV particle release as well. It is hard to identify whether the increase of intracellular nucleocapsid DNA is directly triggered by TIM-1 knockdown or secondary to the change of HBV secretion. The underlying mechanisms of enhanced HBsAg secretion after TIM-1 knockdown also require further investigation. Treating HBV-replicating cells with EIPA led to similar viral DNA and antigen changes. Given that EIPA can also cause alkalinization of endosomes via NHE inhibition, it is possible that the EIPAinduced $\mathrm{pH}$ change in MVBs might affect virus budding and subsequent secretion (65-67). Incubation with anti-LBPA greatly increased HBV DNA from intracellular nucleocapsids and culture medium. Previous research showed that Alix controls LBPA-dependent ILV formation in MVBs (63). Dominant negative Alix can inhibit the secretion of HBV virions via ESCRT disruption in MVBs $(66,68)$. In addition, Alix alone is sufficient for naked capsid release in a nonlytic manner $(68,69)$. Thus, LBPA blockade may not only promote HBV replication but also bring out complex impacts on HBV virion morphogenesis or secretion. Its influence on HBsAg secretion needs further investigation as well. It is possible that HBV release is closely related to exosome entry route, and the relationship between them remains to be interpreted in detail. 
Overall, our results illustrate how receptors, endocytic pathways, and LBPAdependent membrane fusion are exploited by macrophage exosomes to deliver IFN- $\alpha$-induced anti-HBV activities to hepatocytes. This study also highlights the overlap between viruses and exosomes by identifying that the infection strategies of viruses are also applied for exosome entry and exosomal cargo delivery. Dissecting the complete endocytic routes of exosomes may provide a fundamental basis for engineering exosomes as therapeutic vehicles to deliver antiviral molecules with high efficiency.

\section{MATERIALS AND METHODS}

Antibodies, reagents, and chemical inhibitors. Antibodies for LAMP-2 (sc-18822), EEA1 (sc33585), and normal mouse IgG (sc-2025) were purchased from Santa Cruz Biotechnology. Antibodies for Alix (12422-1-AP), TSG101 (14497-1-AP), CD63 (25682-1-AP), RAB5 (11947-1-AP), and RAB7 (55469-1-AP) were purchased from Proteintech Group (Rosemont, IL). Antibody for clathrin heavy chain (ab21679) was from Abcam (Cambridge, MA). Antibodies for $\beta$-actin (A2228) and GFP (G6539) were from Sigma-Aldrich. Antibodies for TIM-1 were from Sigma-Aldrich (SAB1407321) or Novus (NBP1-76701). Antibody for LBPA (MABT837) was from EMD Millipore (Billerica, MA). Fluorescent secondary antibodies (A11001 and A10523) were purchased from Invitrogen. Annexin V -FITC (640905) was purchased from Biolegend (San Diego, CA). Phalloidin-iFluor 488 (23115) was from AAT Bioquest (Sunnyvale, CA). FC-TIM-1-His, a protein of the TIM-1 extracellular domain (AAC39862.1) (Ser 21-Gly 290) which is fused with a polyhistidine tag at the $C$ terminus and the Fc region of human $\operatorname{lgG1}$ at the $\mathrm{N}$ terminus, was from Sino Biological (Beijing, China). Aldehyde-sulfate latex beads ( $4 \%$ [wt/vol], $4 \mu \mathrm{m}$ ) were from Invitrogen (Carlsbad, CA).

Chemical inhibitors, including dynasore (D7693), M $\beta C D$ (C4555), EIPA (A3085), IPA-3 (I2285), and rottlerin (R5648), were from Sigma-Aldrich. Filipin III (70440) was purchased from Cayman Chemical (Ann Arbor, MI). Chlorpromazine (S2456) and nystatin (S1934) were purchased from Selleck Chemicals (Houston, TX).

Cells, liver tissue samples, plasmids, siRNAs, and transfection. The HepG2.2.15, HepG2, LX-2, MGC-803, HEK293T and THP-1 cells used in this study have been described previously $(8,70-73)$. HepG2.2.15, HepG2, LX-2, MGC-803, and HEK293T cells were cultured in Dulbecco modified Eagle medium (DMEM) with 10\% fetal bovine serum (FBS) (Biologic Industries, Beit Haemek, Israel) and penicillin-streptomycin (Invitrogen, Carlsbad, CA), while THP-1 cells were maintained in RPMI 1640 with $10 \%$ FBS and antibiotics. To obtain macrophage-like cells that closely resembled human monocytederived macrophages, THP-1 cells were differentiated via stimulation with phorbol 12-myristate 13acetate (PMA; Sigma-Aldrich, Taufkirchen, Germany), as described previously (8).

Human liver tissue samples were provided by Jian Zhou (Zhongshan Hospital, Shanghai, China). The study protocol conformed to the ethical guidelines of the 1975 Declaration of Helsinki and was approved and supervised by the ethics committee of the Zhongshan Hospital of Fudan University.

Markers of endosomal compartments fused with cyan fluorescent protein (CFP), including CFP-RAB5, CFP-RAB7, and CFP-CD63, were kindly provided by Walther Mothes from Yale University, New Haven, CT $(74,75)$. K44A dynamin-2 pEGFP was a gift from Sandra Schmid (Addgene plasmid 34687). pcDNA3EGFP-Cdc42-T17N (Addgene plasmid 12976) and pcDNA3-EGFP-Rac1-T17N (Addgene plasmid 12982) were gifts from Gary Bokoch. Caveolin 1 labeled with a C-terminal tag of enhanced green fluorescent protein (EGFP) was constructed by insertion of the claveolin-1 CDNA fragment into a pEGFP-N1 expression vector (Clontech, Palo Alto, CA). To produce GFP-carrying exosomes, a THP-1 cell line stably expressing GFP was established via lentivirus transduction. The lentiviral vector PLJM1-GFP (Addgene) was used to generate lentivirus for the transduction, according to the manufacturer's instructions (Addgene). Stable GFP-expressing THP-1 cells were selected by flow cytometric sorting (BD FACSAria II; BD Biosciences, San Jose, CA). Small interfering RNAs (siRNAs) for clathrin heavy chain, caveolin 1, and the negative control were purchased from Santa Cruz Biotechnology. siRNA for TIM-1 was purchased from Ruibo (Guangzhou, China).

DNA plasmid transfection into HepG2 cells was performed using Lipofectamine 2000 (Invitrogen). For RNA-mediated interference, HepG2 cells at $30 \%$ to $40 \%$ confluence were transfected with $50 \mathrm{nM}$ siRNA duplexes designed and purchased from Santa Cruz (Dallas, TX) or Ruibo using RNAiMAX (Invitrogen) according to the manufacturer's instructions. At $24 \mathrm{~h}$ posttransfection, the cells were transfected again with $50 \mathrm{nM}$ concentrations of the same siRNA duplexes. The following treatment was performed $72 \mathrm{~h}$ after the first siRNA transfection.

Exosome purification, characterization, and labeling. Macrophages derived from THP-1 or GFPexpressing THP-1 cells were grown in culture medium supplemented with 10\% FBS (which was depleted of endogenous exosomes by overnight centrifugation at $100,000 \times g$ ). Exosomes from the culture supernatants were isolated by differential centrifugation, as described previously (8). To obtain exosomes from IFN- $\alpha$-treated macrophages, the macrophages were treated for $48 \mathrm{~h}$ with $1,000 \mathrm{U} / \mathrm{ml}$ of IFN- $\alpha$ (PBL Assay Science, New Brunswick, NJ) before isolation. The purified exosomes were characterized via electron microscopy and immunoblot analysis, as described previously (8). Protein amounts of exosomes were quantified using a bicinchoninic acid (BCA) protein assay kit (Pierce, Rockford, IL).

The isolated exosomes were labeled with PKH67 or PKH26 according to the manufacturer's protocol (Sigma-Aldrich) for use in endocytosis assays. For the fluorescence self-quenching assay for membrane 
fusion, R18 (octadecyl rhodamine B chloride; Invitrogen) was inserted into the viral membranes at a self-quenching surface density (40).

Binding and endocytosis assays of exosomes. For the exosome binding assay, GFP-carrying exosomes were preincubated with $1 \mu \mathrm{g} / \mathrm{ml}$ of Fc-TIM-1-His on ice for $40 \mathrm{~min}$. Then the exosomes at the concentration of $20 \mu \mathrm{g} / \mathrm{ml}$ were added to precooled HepG2 cells with plasma membrane stained by PKH26 and allowed to attach for $1 \mathrm{~h}$ on ice $(44,76)$. Unbound exosomes were removed by washing with ice-cold phosphate-buffered saline (PBS).

To assay exosome internalization, 10 to $20 \mu \mathrm{g} / \mathrm{ml}$ of PKH26- or PKH67-labeled exosomes was added to HepG2 cells cultured with serum-free medium and incubated at $37^{\circ} \mathrm{C}$. HepG2 cells were left untreated or pretreated with the desired amounts of inhibitors for $30 \mathrm{~min}$ before incubation with exosomes or endocytic markers. Except cholesterol inhibitors (M $\beta C D$, nystatin, and filipin III), inhibitory compounds were present continuously during subsequent endocytosis assays. Despite moderate cytotoxicity of $\mathrm{M} \beta C D$-treated cells, no significant toxicity was observed for the other inhibitors (data not shown). As controls, HepG2 cells were incubated with $2 \mu \mathrm{g} / \mathrm{ml}$ of Alexa568-transferrin (Invitrogen) or $0.2 \mathrm{mg} / \mathrm{ml}$ of dextran labeled with rhodamine B isothiocyanate (RhoB-dextran; Sigma-Aldrich) for 30 min or $1 \mathrm{~h}$ at $37^{\circ} \mathrm{C}$. For competitive inhibition of TIM-1-mediated exosome entry by Fc-TIM-1-His, HepG2 cells were incubated with PKH26-labeled exosomes in the presence of $1 \mu \mathrm{g} / \mathrm{ml}$ of Fc-TIM-1-His at $37^{\circ} \mathrm{C}$ for $2 \mathrm{~h}$. Endocytosis was stopped, and surface-bound exosomes or markers were removed by washing with ice-cold PBS.

Immunohistochemistry, laser confocal scanning, and time-lapse microscopy. For TIM-1 detection in human liver by immunohistochemistry, slides of human liver tissues were prepared and detected by GServicebio (Wuhan, China).

Confocal images were captured using a Leica TCS SP8 confocal microscope (Leica Microsystems, Buffalo Grove, IL) with a $400 \times$ or $630 \times$ oil objective (pinhole set at 1 Airy unit) and processed using LAS $X$ (Leica).

For time-lapse microscopy analysis, HepG2 cells were grown in 35-mm glass bottom culture dishes with four chambers (Cellvis, Mountain View, CA) overnight. Before microscopic examination, the medium was changed to serum-free DMEM, and fluorescence-labeled exosomes were added and kept in the medium during image collection. Time-lapse images were captured every $10 \mathrm{~min}$ for $6-\mu \mathrm{m}$ slices using a DeltaVision Elite high-resolution microscope (Applied Precision, Issaquah, WA) connected to a $37^{\circ} \mathrm{C}$ incubator and buffered with $5 \% \mathrm{CO}_{2}$. The images were further processed with softWoRx Explorer (Applied Precision) and analyzed with ImageJ (NIH, Bethesda, MD).

For colocalization studies, the distribution patterns of the fluorescent signals were analyzed using the Plot Profile analysis tool of ImageJ, and Pearson's correlation coefficients (Rr) were obtained by using the Colocalization finder plugin of ImageJ. For Pearson's correlation coefficients, the values ranged from 1 (a perfect positive correlation) to -1 (a perfect negative correlation), with 0 representing a random distribution (77). Time-related fluorescence intensities of the R18 dequenching signals were assessed using the Time Series Analyzer V3 plugin of ImageJ.

Flow cytometry analysis. For endocytosis assay, cells were washed three times with ice-cold PBS, detached using trypsin, and subsequently resuspended in PBS with 1\% FBS. Flow cytometry analysis was performed on an LSR Fortessa instrument integrated with the FACSDiva software (BD Biosciences). A minimum of 10,000 events within the gated live cells were collected and analyzed per sample using FlowJo (Tree Star, Ashland, OR).

For PtdSer detection, 4- $\mu \mathrm{m}$ latex beads were coated with exosomes through a 2-h incubation at room temperature. The exosome-bead complexes were then blocked with $200 \mathrm{mM}$ glycine and normal IgG and washed with $1 \%$ FBS, which was followed by annexin V -FITC labeling for 40 min at $4^{\circ} \mathrm{C}$. The exosome-bead complexes were subsequently washed and suspended with $1 \%$ FBS for flow cytometry analysis. A minimum of 50,000 events within the gated exosome-bead complexes were collected and analyzed per sample via FlowJo.

HBV DNA quantitation and antigen measurement. HepG2.2.15 cells pretransfected with siRNAs or pretreated with chemical inhibitors were incubated with exosomes isolated from macrophages with or without IFN- $\alpha$ treatment at a concentration of $10 \mu \mathrm{g} / \mathrm{ml}$ for $48 \mathrm{~h}$. The supernatant of the HepG2.2.15 culture was collected and transferred for viral antigen measurement using the enzyme-linked immunosorbent assay (ELISA; kit from Kehua, Shanghai, China). HBV DNA levels in the culture medium were extracted using a MagNA Pure 96 system (Roche, Shanghai, China). Intracellular core-particle-associated HBV DNA was precipitated and extracted as previously described (70). Viral DNA was quantified by using real-time PCR.

Statistics. All data are presented as the means of duplicates \pm standard deviations (SD). Statistical comparisons were made using a two-tailed Student's $t$ test; $P$ values of 0.05 or less were considered statistically significant.

\section{ACKNOWLEDGMENTS}

We thank Jian Zhou (Zhongshan Hospital affiliated to Fudan University) for providing human liver tissue. We thank Zhigang Yi and Xiaonan Zhang for valuable comments and suggestions and Xiaoting Du, Yaming Li, Ke Qiao, Shuhui Sun for technical assistance.

This work was supported by research grants from the National Natural Science Foundation of China (81471932) National Science and Technology Major Project 
(2018ZX10301202, 2018ZX10301208, 2017ZX10202202), the National Key Research and Development Program of China (2016YFC1200400), the Shanghai Rising-Star Program (14QA1400700), and the Program for Professor of Special Appointment (Eastern Scholar of Shanghai).

\section{REFERENCES}

1. Block TM, Guo H, Guo J-T. 2007. Molecular virology of hepatitis B virus for clinicians. Clin Liver Dis 11:685-706. https://doi.org/10.1016/j.cld .2007.08.002.

2. Shepard CW, Simard EP, Finelli L, Fiore AE, Bell BP. 2006. Hepatitis B virus infection: epidemiology and vaccination. Epidemiol Rev 28:112-125. https://doi.org/10.1093/epirev/mxj009.

3. Levrero M, Zucman-Rossi J. 2016. Mechanisms of HBV-induced hepatocellular carcinoma. J Hepatol 64:S84-S101. https://doi.org/10.1016/j.jhep.2016 .02 .021 .

4. Loggi E, Vitale G, Conti F, Bernardi M, Andreone P. 2015. Chronic hepatitis B: are we close to a cure? Dig Liver Dis 47:836-841. https:// doi.org/10.1016/j.dld.2015.05.019.

5. Chen J, Wu M, Zhang X, Zhang W, Zhang Z, Chen L, He J, Zheng Y, Chen C, Wang F, Hu Y, Zhou X, Wang C, Xu Y, Lu M, Yuan Z. 2013. Hepatitis B virus polymerase impairs interferon-alpha-induced STA $T$ activation through inhibition of importin-alpha5 and protein kinase C-delta. Hepatology 57:470-482. https://doi.org/10.1002/hep.26064.

6. Lutgehetmann M, Bornscheuer T, Volz T, Allweiss L, Bockmann JH, Pollok JM, Lohse AW, Petersen J, Dandri M. 2011. Hepatitis B virus limits response of human hepatocytes to interferon-alpha in chimeric mice. Gastroenterology 140:2074-2083.e2. https://doi.org/10.1053/j .gastro.2011.02.057.

7. Rehermann B, Bertoletti A. 2015. Immunological aspects of antiviral therapy of chronic hepatitis $B$ virus and hepatitis $C$ virus infections. Hepatology 61:712-721. https://doi.org/10.1002/hep.27323.

8. Li J, Liu K, Liu Y, Xu Y, Zhang F, Yang H, Liu J, Pan T, Chen J, Wu M, Zhou $X$, Yuan Z. 2013. Exosomes mediate the cell-to-cell transmission of IFN-alpha-induced antiviral activity. Nat Immunol 14:793-803. https:// doi.org/10.1038/ni.2647.

9. Giugliano S, Kriss M, Golden-Mason L, Dobrinskikh E, Stone AEL, Soto-Gutierrez A, Mitchell A, Khetani SR, Yamane D, Stoddard M, Li H, Shaw GM, Edwards MG, Lemon SM, Gale M, Shah VH, Rosen HR. 2015. $\mathrm{HCV}$ infection induces autocrine interferon signaling by human liver endothelial cell and release of exosomes, which inhibits viral replication. Gastroenterology 148:392-402.e313. https://doi.org/10.1053/ j.gastro.2014.10.040.

10. van Dongen HM, Masoumi N, Witwer KW, Pegtel DM. 2016. Extracellular vesicles exploit viral entry routes for cargo delivery. Microbiol Mol Biol Rev 80:369-386. https://doi.org/10.1128/MMBR.00063-15.

11. Klenerman P, Ramamurthy N. 2015. Liver sinusoidal endothelial cells: an antiviral "defendothelium." Gastroenterology 148:288-291. https://doi .org/10.1053/j.gastro.2014.12.010.

12. Bordon Y. 2013. Viral immunity: arms convoy. Nat Rev Immunol 13:547. https://doi.org/10.1038/nri3500.

13. Thery C, Ostrowski M, Segura E. 2009. Membrane vesicles as conveyors of immune responses. Nat Rev Immunol 9:581-593. https://doi.org/10 $.1038 /$ nri2567.

14. Izquierdo-Useros N, Puertas MC, Borras FE, Blanco J, Martinez-Picado J. 2011. Exosomes and retroviruses: the chicken or the egg? Cell Microbiol 13:10-17. https://doi.org/10.1111/j.1462-5822.2010.01542.x.

15. Pegtel DM, Cosmopoulos K, Thorley-Lawson DA, van Eijndhoven MAJ, Hopmans ES, Lindenberg JL, de Gruijl TD, Würdinger T, Middeldorp JM. 2010. Functional delivery of viral miRNAs via exosomes. Proc Natl Acad Sci U S A 107:6328-6333. https://doi.org/10.1073/pnas.0914843107.

16. Schorey JS, Bhatnagar S. 2008. Exosome function: from tumor immunology to pathogen biology. Traffic 9:871-881. https://doi.org/10.1111/j .1600-0854.2008.00734.x.

17. Robbins PD, Morelli AE. 2014. Regulation of immune responses by extracellular vesicles. Nat Rev Immunol 14:195-208. https://doi.org/10 .1038/nri3622

18. Lai RC, Yeo RW, Tan KH, Lim SK. 2013. Exosomes for drug delivery-a novel application for the mesenchymal stem cell. Biotechnol Adv 31: 543-551. https://doi.org/10.1016/j.biotechadv.2012.08.008.

19. van den Boorn JG, Schlee M, Coch C, Hartmann G. 2011. SiRNA delivery with exosome nanoparticles. Nat Biotechnol 29:325. https://doi.org/10 $.1038 / \mathrm{nbt} .1830$

20. Wei X, Liu C, Wang H, Wang L, Xiao F, Guo Z, Zhang H. 2016. Surface phosphatidylserine is responsible for the internalization on $\mathrm{mi}$ crovesicles derived from hypoxia-induced human bone marrow mesenchymal stem cells into human endothelial cells. PLoS One 11:e0147360. https://doi.org/10.1371/journal.pone.0147360.

21. Morelli AE, Larregina AT, Shufesky WJ, Sullivan ML, Stolz DB, Papworth GD, Zahorchak AF, Logar AJ, Wang Z, Watkins SC, Falo LD, Jr, Thomson AW. 2004. Endocytosis, intracellular sorting, and processing of exosomes by dendritic cells. Blood 104:3257-3266. https://doi.org/10.1182/blood -2004-03-0824.

22. Christianson HC, Svensson KJ, van Kuppevelt TH, Li JP, Belting M. 2013. Cancer cell exosomes depend on cell-surface heparan sulfate proteoglycans for their internalization and functional activity. Proc Natl Acad Sci U S A 110:17380-17385. https://doi.org/10.1073/pnas .1304266110 .

23. Tian T, Zhu YL, Zhou YY, Liang GF, Wang YY, Hu FH, Xiao ZD. 2014. Exosome uptake through clathrin-mediated endocytosis and macropinocytosis and mediating miR-21 delivery. J Biol Chem 289:22258-22267. https://doi.org/10.1074/jbc.M114.588046.

24. Nanbo A, Kawanishi E, Yoshida R, Yoshiyama H. 2013. Exosomes derived from Epstein-Barr virus-infected cells are internalized via caveoladependent endocytosis and promote phenotypic modulation in target cells. J Virol 87:10334-10347. https://doi.org/10.1128/JVI.01310-13.

25. Yao Z, Qiao Y, Li X, Chen J, Ding J, Bai L, Shen F, Shi B, Liu J, Peng L, Li J, Yuan Z. 2018. Exosomes exploit the virus entry machinery and pathway to transmit alpha interferon-induced antiviral activity. bioRxiv https://doi.org/10.1101/300715

26. Fitzner D, Schnaars M, van Rossum D, Krishnamoorthy G, Dibaj P, Bakhti M, Regen T, Hanisch UK, Simons M. 2011. Selective transfer of exosomes from oligodendrocytes to microglia by macropinocytosis. J Cell Sci 124:447-458. https://doi.org/10.1242/jcs.074088.

27. Amara A, Mercer J. 2015. Viral apoptotic mimicry. Nat Rev Microbiol 13:461-469. https://doi.org/10.1038/nrmicro3469.

28. Moller-Tank S, Albritton LM, Rennert PD, Maury W. 2014. Characterizing functional domains for TIM-mediated enveloped virus entry. J Virol 88:6702-6713. https://doi.org/10.1128/JVI.00300-14.

29. Mulcahy LA, Pink RC, Carter DR. 2014. Routes and mechanisms of extracellular vesicle uptake. J Extracell Vesicles 3:1. https://doi.org/10 .3402/jev.v3.24641.

30. Doherty GJ, McMahon HT. 2009. Mechanisms of endocytosis. Annu Rev Biochem 78:857-902. https://doi.org/10.1146/annurev.biochem .78.081307.110540.

31. Nonnenmacher M, Weber T. 2011. Adeno-associated virus 2 infection requires endocytosis through the CLIC/GEEC pathway. Cell Host Microbe 10:563-576. https://doi.org/10.1016/j.chom.2011.10.014.

32. Antonny B, Burd C, De Camilli P, Chen E, Daumke O, Faelber K, Ford M, Frolov VA, Frost A, Hinshaw JE, Kirchhausen T, Kozlov MM, Lenz M, Low $\mathrm{HH}$, McMahon H, Merrifield C, Pollard TD, Robinson PJ, Roux A, Schmid S. 2016. Membrane fission by dynamin: what we know and what we need to know. EMBO J 35:2270-2284. https://doi.org/10.15252/embj .201694613

33. Mercer J, Helenius A. 2012. Gulping rather than sipping: macropinocytosis as a way of virus entry. Curr Opin Microbiol 15:490-499. https:// doi.org/10.1016/j.mib.2012.05.016.

34. Subtil A, Gaidarov I, Kobylarz K, Lampson MA, Keen JH, McGraw TE. 1999. Acute cholesterol depletion inhibits clathrin-coated pit budding. Proc Natl Acad Sci U S A 96:6775-6780. https://doi.org/10.1073/pnas.96.12 .6775 .

35. Mercer J, Helenius A. 2009. Virus entry by macropinocytosis. Nat Cell Biol 11:510-520. https://doi.org/10.1038/ncb0509-510.

36. Branza-Nichita N, Macovei A, Lazar C. 2012. Caveolae-dependent endocytosis in viral infection. In Cereza B (ed), Molecular regulation of endo- 
cytosis. IntechOpen, London, United Kingdom. https://doi.org/10.5772/ 2989.

37. Cokakli M, Erdal E, Nart D, Yilmaz F, Sagol O, Kilic M, Karademir S, Atabey N. 2009. Differential expression of Caveolin-1 in hepatocellular carcinoma: correlation with differentiation state, motility and invasion. BMC Cancer 9:65. https://doi.org/10.1186/1471-2407-9-65.

38. Mercer J, Schelhaas M, Helenius A. 2010. Virus entry by endocytosis. Annu Rev Biochem 79:803-833. https://doi.org/10.1146/annurev -biochem-060208-104626.

39. Koivusalo M, Welch C, Hayashi H, Scott CC, Kim M, Alexander T, Touret N, Hahn KM, Grinstein S. 2010. Amiloride inhibits macropinocytosis by lowering submembranous $\mathrm{pH}$ and preventing Rac1 and Cdc42 signaling. J Cell Physiol 188:547-563. https://doi.org/10.1083/jcb.200908086.

40. Nour AM, Li Y, Wolenski J, Modis Y. 2013. Viral membrane fusion and nucleocapsid delivery into the cytoplasm are distinct events in some flaviviruses. PLoS Pathog 9:e1003585. https://doi.org/10.1371/journal .ppat.1003585.

41. Le Blanc I, Luyet PP, Pons V, Ferguson C, Emans N, Petiot A, Mayran N, Demaurex N, Faure J, Sadoul R, Parton RG, Gruenberg J. 2005. Endosome-to-cytosol transport of viral nucleocapsids. Nat Cell Biol 7:653-664. https://doi.org/10.1038/ncb1269.

42. Erazo-Oliveras A, Najjar K, Truong D, Wang TY, Brock DJ, Prater AR, Pellois JP. 2016. The late endosome and its lipid BMP act as gateways for efficient cytosolic access of the delivery agent dfTAT and its macromolecular cargos. Cell Chem Biol 23:598-607. https://doi.org/ 10.1016/j.chembiol.2016.03.016.

43. Wang S, Sun H, Tanowitz M, Liang X-H, Crooke ST. 2017. Intra-endosomal trafficking mediated by lysobisphosphatidic acid contributes to intracellular release of phosphorothioate-modified antisense oligonucleotides. Nucleic Acids Res 45:5309-5322. https://doi.org/10.1093/nar/gkx231.

44. Citovsky V, Blumenthal R, Loyter A. 1985. Fusion of Sendai virions with phosphatidylcholine-cholesterol liposomes reflects the viral activity required for fusion with biological membranes. FEBS Lett 193:135-140. https://doi.org/10.1016/0014-5793(85)80137-X.

45. White JM, Whittaker GR. 2016. Fusion of enveloped viruses in endosomes. Traffic 17:593-614. https://doi.org/10.1111/tra.12389.

46. Roth SL, Whittaker GR. 2011. Promotion of vesicular stomatitis virus fusion by the endosome-specific phospholipid bis(monoacylglycero)phosphate (BMP). FEBS Lett 585:865-869. https://doi.org/10.1016/j febslet.2011.02.015.

47. Farzan M, Pasqual G, Rojek JM, Masin M, Chatton J-Y, Kunz S. 2011. Old World arenaviruses enter the host cell via the multivesicular body and depend on the endosomal sorting complex required for transport. PLoS Pathog 7:e1002232. https://doi.org/10.1371/journal.ppat.1002232.

48. Thery C, Zitvogel L, Amigorena S. 2002. Exosomes: composition, biogenesis and function. Nat Rev Immunol 2:569-579. https://doi.org/10.1038/ nri855.

49. Kaplan G, Totsuka A, Thompson P, Akatsuka T, Moritsugu Y, Feinstone SM. 1996. Identification of a surface glycoprotein on African green monkey kidney cells as a receptor for hepatitis A virus. EMBO J 15: 4282-4296. https://doi.org/10.1002/j.1460-2075.1996.tb00803.x.

50. Ramakrishnaiah V, van der Laan LJW. 2014. Hepatitis virus hijacks shuttle: exosome-like vesicles provide protection against neutralizing antibodies. Hepatology 59:2416-2418. https://doi.org/10.1002/hep.26943.

51. Naslund TI, Paquin-Proulx D, Paredes PT, Vallhov H, Sandberg JK, Gabrielsson S. 2014. Exosomes from breast milk inhibit HIV-1 infection of dendritic cells and subsequent viral transfer to CD4+ T cells. AIDS 28:171-180. https://doi.org/10.1097/QAD.0000000000000159.

52. Hazan-Halevy I, Rosenblum D, Weinstein S, Bairey O, Raanani P, Peer D. 2015. Cell-specific uptake of mantle cell lymphoma-derived exosomes by malignant and non-malignant B-lymphocytes. Cancer Lett 364: 59-69. https://doi.org/10.1016/j.canlet.2015.04.026.

53. Zech D, Rana S, Büchler MW, Zöller M. 2012. Tumor-exosomes and leukocyte activation: an ambivalent crosstalk. Cell Commun Signal 10:37. https://doi.org/10.1186/1478-811X-10-37.

54. Rejman J, Oberle V, Zuhorn IS, Hoekstra D. 2004. Size-dependent internalization of particles via the pathways of clathrin- and caveolaemediated endocytosis. Biochem J 377:159-169. https://doi.org/10.1042/ bj20031253.

55. Feng D, Zhao WL, Ye YY, Bai XC, Liu RQ, Chang LF, Zhou Q, Sui SF. 2010. Cellular internalization of exosomes occurs through phagocytosis. Traffic 11:675-687. https://doi.org/10.1111/j.1600-0854.2010.01041.x.

56. McKelvey KJ, Powell KL, Ashton AW, Morris JM, McCracken SA. 2015.
Exosomes: mechanisms of uptake. J Circ Biomark 4:7. https://doi.org/10 $.5772 / 61186$.

57. Parolini I, Federici C, Raggi C, Lugini L, Palleschi S, De Milito A, Coscia C, lessi E, Logozzi M, Molinari A, Colone M, Tatti M, Sargiacomo M, Fais S. 2009. Microenvironmental $\mathrm{pH}$ is a key factor for exosome traffic in tumor cells. J Biol Chem 284:34211-34222. https://doi.org/10.1074/jbc.M109 .041152 .

58. Pekosz A, de Vries $E$, Tscherne DM, Wienholts MJ, Cobos-Jiménez V Scholte F, García-Sastre A, Rottier PJM, de Haan CAM. 2011. Dissection of the influenza $A$ virus endocytic routes reveals macropinocytosis as an alternative entry pathway. PLoS Pathog 7:e1001329. https://doi.org/10 .1371/journal.ppat.1001329.

59. Mettlen M, Platek A, Van Der Smissen $P$, Carpentier $S$, Amyere $M$, Lanzetti L, de Diesbach P, Tyteca D, Courtoy PJ. 2006. Src triggers circular ruffling and macropinocytosis at the apical surface of polarized MDCK cells. Traffic 7:589-603. https://doi.org/10.1111/j.1600 -0854.2006.00412.x.

60. Piper RC, Katzmann DJ. 2007. Biogenesis and function of multivesicular bodies. Annu Rev Cell Dev Biol 23:519-547. https://doi.org/10.1146/ annurev.cellbio.23.090506.123319.

61. Matos PM, Marin M, Ahn B, Lam W, Santos NC, Melikyan GB. 2013. Anionic lipids are required for vesicular stomatitis virus $G$ proteinmediated single particle fusion with supported lipid bilayers. J Biol Chem 288:12416-12425. https://doi.org/10.1074/jbc.M113.462028.

62. Zaitseva E, Yang ST, Melikov K, Pourmal S, Chernomordik LV. 2010. Dengue virus ensures its fusion in late endosomes using compartmentspecific lipids. PLoS Pathog 6:e1001131. https://doi.org/10.1371/journal .ppat.1001131.

63. Matsuo H, Chevallier J, Mayran N, Le Blanc I, Ferguson C, Faure J, Blanc NS, Matile S, Dubochet J, Sadoul R, Parton RG, Vilbois F, Gruenberg J. 2004. Role of LBPA and Alix in multivesicular liposome formation and endosome organization. Science 303:531-534. https://doi.org/10.1126/ science. 1092425.

64. Li M, Ablan SD, Miao C, Zheng YM, Fuller MS, Rennert PD, Maury W, Johnson MC, Freed EO, Liu SL. 2014. TIM-family proteins inhibit HIV-1 release. Proc Natl Acad Sci U S A 111:E3699-E3707. https://doi.org/10 $.1073 /$ pnas. 1404851111.

65. Lambert C, Doring T, Prange R. 2007. Hepatitis B virus maturation is sensitive to functional inhibition of ESCRT-III, Vps4, and gamma 2-adaptin. J Virol 81:9050-9060. https://doi.org/10.1128/JVl.00479-07.

66. Watanabe T, Sorensen EM, Naito A, Schott M, Kim S, Ahlquist P. 2007. Involvement of host cellular multivesicular body functions in hepatitis $B$ virus budding. Proc Natl Acad Sci U S A 104:10205-10210. https://doi .org/10.1073/pnas.0704000104.

67. Gekle M, Drumm K, Mildenberger S, Freudinger R, Gaßner B, Silbernagl S. 1999. Inhibition of $\mathrm{Na}(+)-\mathrm{H}(+)$ exchange impairs receptor-mediated albumin endocytosis in renal proximal tubule-derived epithelial cells from opossum. J Physiol 520:709-721. https://doi.org/10.1111/j.1469 -7793.1999.00709.x.

68. Prange R. 2012. Host factors involved in hepatitis B virus maturation, assembly, and egress. Med Microbiol Immunol 201:449-461. https://doi .org/10.1007/s00430-012-0267-9.

69. Bardens A, Doring T, Stieler J, Prange R. 2011. Alix regulates egress of hepatitis B virus naked capsid particles in an ESCRT-independent manner. Cell Microbiol 13:602-619. https://doi.org/10.1111/j.1462-5822.2010 .01557.x.

70. Li J, Lin S, Chen Q, Peng L, Zhai J, Liu Y, Yuan Z. 2010. Inhibition of hepatitis $B$ virus replication by MyD88 involves accelerated degradation of pregenomic RNA and nuclear retention of pre-S/S RNAs. J Virol 84:6387-6399. https://doi.org/10.1128/JVI.00236-10.

71. Liu Y, Li J, Chen J, Li Y, Wang W, Du X, Song W, Zhang W, Lin L, Yuan Z. 2015. Hepatitis B virus polymerase disrupts K63-linked ubiquitination of STING to block innate cytosolic DNA-sensing pathways. J Virol 89: 2287-2300. https://doi.org/10.1128/JVI.02760-14.

72. Xu L, Hui AY, Albanis E, Arthur MJ, O'Byrne SM, Blaner WS, Mukherjee $P$, Friedman SL, Eng FJ. 2005. Human hepatic stellate cell lines, LX-1 and LX-2: new tools for analysis of hepatic fibrosis. Gut 54:142-151. https:// doi.org/10.1136/gut.2004.042127.

73. Yao K, Wang W, Li H, Lin J, Tan W, Chen Y, Guo L, Lin D, Chen T, Zhou J, Zhai S, Yu W. 2018. Bacillus Calmette Guerin (BCG) activates lymphocyte to promote autophagy and apoptosis of gastric cancer MGC-803 cell. Cell Mol Biol (Noisy-le-grand) 64:11-16. https://doi.org/10.14715/ $\mathrm{cmb} / 2018.64 .6 .3$.

74. Sherer NM, Lehmann MJ, Jimenez-Soto LF, Ingmundson A, Horner SM, 
Cicchetti G, Allen PG, Pypaert M, Cunningham JM, Mothes W. 2003. Visualization of retroviral replication in living cells reveals budding into multivesicular bodies. Traffic 4:785-801. https://doi.org/10.1034/j.1600 -0854.2003.00135.x.

75. Auwerx J. 1991. The human leukemia cell line, THP-1: a multifacetted model for the study of monocyte-macrophage differentiation. Experientia 47:22-31. https://doi.org/10.1007/BF02041244.
76. Lee GM, Fong S, Francis K, Oh DJ, Palsson BO. 2000. In situ labeling of adherent cells with PKH26. In Vitro Cell Dev Biol Anim 36:4-6. https:// doi.org/10.1290/1071-2690(2000)036<0004:ISLOAC>2.0.CO;2.

77. Barlow AL, Macleod A, Noppen S, Sanderson J, Guérin C. 2010. Colocalization analysis in fluorescence micrographs: verification of a more accurate calculation of Pearson's correlation coefficient. Microsc Microanal 16:710-724. https://doi.org/10.1017/S143192761009389X. 